\title{
Autonomous Coordinated Control of the Light Guide for Positioning in Vitreoretinal Surgery
}

\author{
Yuki Koyama, Murilo M. Marinho, Mamoru Mitsuishi, and Kanako Harada
}

\begin{abstract}
Vitreoretinal surgery is challenging even for expert surgeons owing to the delicate target tissues and the diminutive workspace in the retina. In addition to improved dexterity and accuracy, robot assistance allows for (partial) task automation. In this work, we propose a strategy to automate the motion of the light guide with respect to the surgical instrument. This automation allows the instrument's shadow to always be inside the microscopic view, which is an important cue for the accurate positioning of the instrument in the retina. We show simulations and experiments demonstrating that the proposed strategy is effective in a 700-point grid in the retina of a surgical phantom. Furthermore, we integrated the proposed strategy with image processing and succeeded in positioning the surgical instrument's tip in the retina, relying on only the robot's geometric information and microscopic images.
\end{abstract}

Index Terms-Medical Robots and Systems, Kinematics, Collision Avoidance.

\section{INTRODUCTION}

V ITREORETINAL minimally invasive surgical procedures are routinely conducted manually by surgeons. The diminutive workspace in the retina is viewed through an ophthalmic microscope. In one hand, surgeons hold a surgical instrument, such as $30 \mathrm{~mm}$ long forceps with $1 \mathrm{~mm}$ diameter. In their other hand, surgeons hold a small lantern, called a light guide, to illuminate the workspace in the eyeball. In some cases, a chandelier endoilluminator ${ }^{1}$ or the light provided from the microscope are used.

Among challenging vitreoretinal procedures, two of them stand out: the peeling of the $2.5 \mu \mathrm{m}$ thick inner limiting membrane and the cannulation of retinal blood vessels smaller than $100 \mu \mathrm{m}$ in diameter. The average amplitude of hand tremors is approximately $100 \mu \mathrm{m}$ [1] and considerably higher than the accuracy needed for these tasks. In this sense, these tasks can be considered difficult to perform manually, even for experienced surgeons.

In this context, several robotic systems have been developed to enhance operational accuracy and increase patient safety [2]. These systems are exclusively made for eye surgery and can be classified into three main categories: hand-held robotic

This research was funded in part by the New Energy and Industrial Technology Development Organization (Japan), in part by the ImPACT Program of the Council for Science, Technology and Innovation (Cabinet Office, Government of Japan), and in part by the Mori Manufacturing Research and Technology Foundation.

(Corresponding author: Murilo M. Marinho)

The authors are with the Department of Mechanical Engineering, the University of Tokyo, Tokyo, Japan. Emails: \{yuuki-koyama581, murilo, mamoru, kanakoharada\}@g.ecc.u-tokyo.ac.jp.

${ }^{1} \mathrm{~A}$ chandelier illuminator is a light source attached to the wall of the eye and spreads the light in a wider angle. devices [3], [4], cooperatively-controlled systems [5], [6], and tele-operated systems [7]-[10].

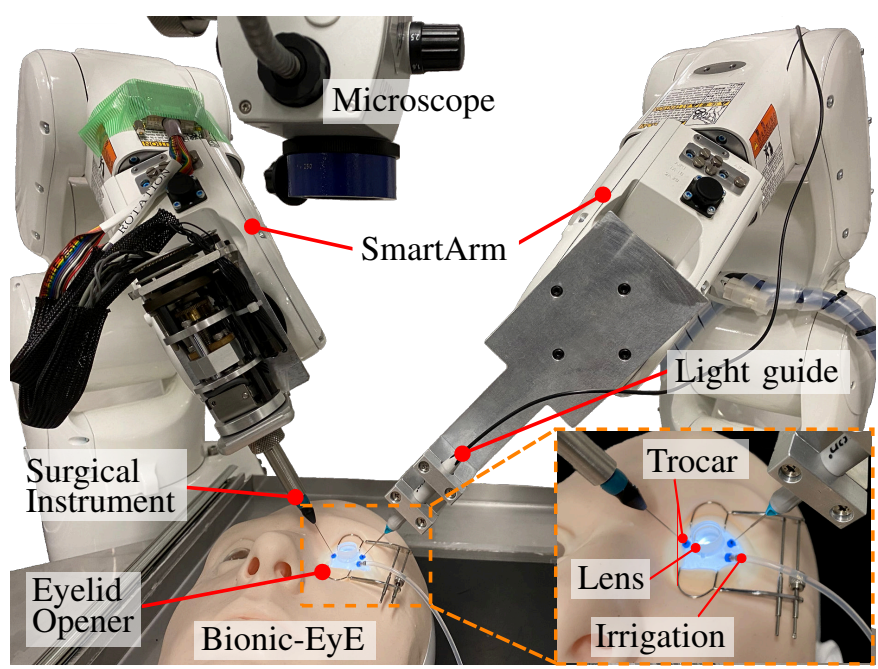

Fig. 1. The SmartArm [11] robotic system outfitted for vitreoretinal surgery. The left manipulator holds a surgical instrument to treat tissues, and the right manipulator holds a light guide to illuminate the workspace in the eyeball. The vitreoretinal surgical phantom (Bionic-EyE) [12] is placed between the two robots.

In a different approach to having a robotic system exclusively for eye surgical procedures, our group has been working on the tele-operated SmartArm surgical robotic system. Unlike other tele-operated systems, the SmartArm system does not have mechanical constraints for a particular task. This versatility has allowed us to preliminarily validate the SmartArm in-vitro in multiple types of surgery in constrained workspaces. For instance, endonasal surgery [11] and pediatric surgery [13], [14]. In another prior work, we have preliminarily established that the SmartArm can achieve accuracy within the required order of magnitude for eye surgical procedures, which is about a few dozen micrometers depending on the task [15]. The average trajectory tracing accuracy of the SmartArm was $22 \mu \mathrm{m}$ and considerably better than the recorded precision of a surgeon, which was $108 \mu m$ [1]. The SmartArm, equipped for vitreoretinal surgery, is shown in Fig. 1.

Robotic systems can provide increased dexterity and accuracy and are designed to enact the motion generated by the surgeon directly. (Semi-)automation can further improve the safety and efficiency of robot-aided surgical procedures.

To automate vitreoretinal tasks, accurate and safe positioning of the surgical instrument's tip on the retina is fundamental. One particular difficulty of retinal positioning is the estimation of the distance between the tip of the surgical instrument and 
the retina. The reduced size of the workspace, small parallax in stereo microscopes, and the optical aberrations caused by the vitreous body make regular image processing algorithms unsuitable. Some groups address this difficulty by integrating optical coherence tomography (OCT) into their systems to estimate the instrument's position [16], [17]. However, OCT is high cost, requires an extended processing time, and has a limited scan range. Some groups have tackled these drawbacks of OCT, and Sommersperger et al. [18] achieved real-time estimation of the tool to layer distance employing 4D OCT. Nonetheless, those systems are not yet easily available.

In a different approach, our group has been investigating the use of the instrument's shadow to estimate the proximity between the instrument's tip and the retina during instrument positioning [19]. The rationale behind this idea is that the distance between the instrument's tip and its shadow and the distance between the instrument's tip and the retina are proportional related. Therefore, by correctly angling the lighting instrument, we can use the geometric relationship among those objects to judge if the positioning has been completed. As a lighting instrument, we use the light guide given that it allows for some freedom in controlling the direction of the light.

For this approach to be successful, it is important to ensure that the tip of the instrument's shadow is visible in the microscopic image during the positioning process. With this motivation, in this work, we focus on autonomous bimanual control of the light guide and the instrument in a further step toward vitreoretinal surgery automation.

\section{A. Related works}

Other groups have also addressed the estimation of the distance or proximity between the surgical instrument's tip and the retina. For instance, Richa et al. [20] used stereo images and detected the proximity based on the relative stereo disparity. Bergeless et al. [21], [22] presented a wide-angle localization method for microrobotic devices using an optical system. The team of Carnegie Mellon University [23], [24] proposed a retinal surface estimation method using projected beam patterns on the retinal surface. In recent research, Kim et al. [25] used distinct tool-shadow dynamics as cues to train their network to automate a tool-navigation task. Zhou et al. [26] demonstrated a novel method for 3D guidance of the instrument based on the projection mechanism of a spotlight integrated into the instrument.

Some groups have studied the automation of a single instrument in eye surgery procedures [27], [28]. Other groups have studied bimanual control. Wei et al. [29] evaluated the coupled kinematics of two or more manipulators in hollow suspended organs, e.g., the eye. More recently, He et al. [30] proposed an automatic light pipe actuation system and succeeded in autonomously aligning the light guide with the target point on the retina.

Existing strategies are not compatible with our shadowbased autonomous positioning approach [19]. In this work, our interest lies in finding a control strategy that guarantees the visibility of the shadow of the instrument tip during autonomous positioning. We propose the use of the dynamic regional virtual fixtures generated by vector-field inequalities (VFIs) [31]. Some groups also introduced virtual fixture schemes for robot-aided vitreoretinal surgery [32], [33]. However, their method needs to artificially reduce the degrees of freedom to enforce the constraints, and only the constraints around the insertion point are applied. On the other hand, the VFI method allows us to satisfy all of the proposed constraints without reducing the degrees of freedom. This is an important aspect because using all degrees-of-freedom available for the task incurs, in general, better dexterity. Moreover, this makes our method practical and independent of a particular robot's geometry.

The proposed VFIs satisfy all the constraints for vitreoretinal surgery and autonomously move the light guide dynamically with respect to changes in the position of the surgical instrument with safety guaranteed by design.

\section{B. Statement of contributions}

The main contributions of this work are: (1) a novel threestep coordinated control algorithm for two robotic manipulators aiming shadow-based autonomous retinal positioning; (2) new vitreoretinal-surgery-specific dynamic regional virtual fixtures generated by VFIs (Section IX); (3) experimental results that demonstrate the real-world feasibility of our shadowbased autonomous positioning strategy considering, even, its integration with an image-processing algorithm.

\section{Problem Statement}

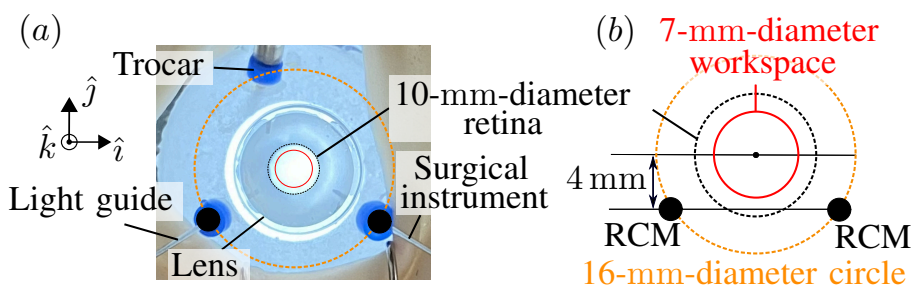

Fig. 2. The definition of the workspace and the location of the RCM. (a) shows the 10 -mm-diameter retina inside the eye phantom which appears smaller because of the disposable flat lens. The lens has a focal point compatible with the microscope. (b) shows the definition of the workspace and the RCM points, which correspond to the trocar points.

Consider the setup shown in Fig. 1. Let R1 be the robot holding the surgical instrument, with joint values $\boldsymbol{q}_{\mathrm{R} 1} \in \mathbb{R}^{n_{1}}$, and $\mathrm{R} 2$ be the robot holding the light guide, with joint values $\boldsymbol{q}_{\mathrm{R} 2} \in \mathbb{R}^{n_{2}}$. The instruments are inserted into a vitreoretinal surgical phantom (Bionic-EyE [12]) placed between the two robotic arms. The eye phantom has a $10-\mathrm{mm}$-diameter retina, as shown in Fig. 2-(a). Similar to manual surgery, a disposable flat lens is added to provide the correct view of the workspace. In this work, the size of the workspace was defined as a 7-mmdiameter circular region in the retina based on the discussion with partner expert surgeons [34], as shown in Fig. 2-(b). 2D images of the workspace are obtained through an ophthalmic microscope placed above the eye phantom.

Fig. 2-(b) shows the location of the remote center-of-motion (RCM), which must be generated by the robot to match the insertion point in the patient. The RCM points correspond to 
the trocar points. As in manual surgery, there is some freedom in the placement of the insertion points. We set the insertion points below the centerline of the eye for better visibility of the instrument and its shadow.

\section{A. Goal}

Our goal is to autonomously position the instrument at a point in the retina using the instrument's shadow as supporting information. In this work, we propose a reliable control strategy to enact the envisioned shadow-based retinal positioning approach. Our focus on this work is the development of the control strategy itself. We integrate our strategy with image processing for a proof-of-concept validation, but further progress in that direction is left as a topic for future work.

\section{B. Premises}

Our robot controller was designed under the following premises:

- The robots are velocity-controlled by the signals $\boldsymbol{u}_{\mathrm{R} 1} \triangleq$ $\dot{\boldsymbol{q}}_{\mathrm{R} 1} \in \mathbb{R}^{n_{1}}$ and $\boldsymbol{u}_{\mathrm{R} 2} \triangleq \dot{\boldsymbol{q}}_{\mathrm{R} 2} \in \mathbb{R}^{n_{2}}$ (Premise I).

- The solution $\boldsymbol{u}_{\mathrm{R} 1}=\mathbf{0}_{n_{1}}$ and $\boldsymbol{u}_{\mathrm{R} 2}=\mathbf{0}_{n_{2}}$ must always be feasible. That is, at worst the robots can stop moving (Premise II).

- All constraints (summarized in Section II-C and Section II-D) must be satisfied at the beginning of the autonomous motion $(t=0)$ (Premise III).

- The robots have a kinematic model that is precise enough for the task. (Premise IV).

Regarding Premise IV, we have shown in prior work in highly constrained workspaces that this is a reasonable assumption in terms of safety [31]. In terms of precise positioning in the retina, we have to rely on the microscopic image for increased accuracy, as we preliminarily do in the experimental section of this work. In parallel to this work, we are also investigating online-calibration strategies to adjust the robot's kinematic model online [35].

\section{Robot-assisted vitreoretinal task constraints}

A robotic system has to impose the following constraints to safely conduct vitreoretinal tasks:

- The shafts of the instruments must always pass through their respective insertion points (RCM) in the eye $\left(C_{R}\right)$.

- The light guide's tip must never touch the retina $\left(C_{r}\right)$.

- The shafts of the instruments must never collide with each other $\left(\mathrm{C}_{\mathrm{s}}\right)$.

- The tips of the instruments must always remain inside the eye $\left(\mathrm{C}_{\text {tr }}\right)$.

- The robots must never collide with the microscope $\left(\mathrm{C}_{\mathrm{m}}\right)$ and each other $\left(\mathrm{C}_{\mathrm{ro}}\right)$.

- The joint values must never exceed their limits $\left(C_{j}\right)$.

\section{Shadow-based autonomous positioning constraints}

For the shadow-based positioning to guarantee the visibility of the shadow, we have three additional constraints:

- The tip of the shadow of the instrument must always be visible in the microscopic view $\left(\mathrm{C}_{1}\right)$.
- The tip of the instrument must be illuminated sufficiently so that the contour of its shadow can be visible $\left(\mathrm{C}_{\mathrm{t}}\right)$ at all times $\left(\mathrm{C}_{2}\right)$.

\section{MATHEMATICAL BACKGROUND}

In this section, we summarize the required mathematical background for understanding the proposed controller.

\section{A. Quaternions and operators}

The quaternion set is

$$
\mathbb{H} \triangleq\left\{h_{1}+\hat{\imath} h_{2}+\hat{\jmath} h_{3}+\hat{k} h_{4}: h_{1}, h_{2}, h_{3}, h_{4} \in \mathbb{R}\right\},
$$

in which the imaginary units $\hat{\imath}, \hat{\jmath}$, and $\hat{k}$ have the following properties: $\hat{\imath}^{2}=\hat{\jmath}^{2}=\hat{k}^{2}=\hat{\imath} \hat{\jmath} \hat{k}=-1$. Elements of the set $\mathbb{H}_{p} \triangleq\{\boldsymbol{h} \in \mathbb{H}: \operatorname{Re}(\boldsymbol{h})=0\}$ represent points in $\mathbb{R}^{3}$. The set of quaternions with unit norm, $\mathbb{S}^{3} \triangleq\{\boldsymbol{r} \in \mathbb{H}:\|\boldsymbol{r}\|=1\}$, represent the rotation $\boldsymbol{r}=\cos (\phi / 2)+\boldsymbol{v} \sin (\phi / 2)$, where $\phi \in \mathbb{R}$ is the rotation angle around the rotation axis $\boldsymbol{v} \in \mathbb{S}^{3} \cap \mathbb{H}_{p}$.

The operator $\operatorname{vec}_{4}$ maps a quaternion $\boldsymbol{h} \in \mathbb{H}$ into a column vector $\mathbb{R}^{4}$. We use a shorthand version $\mathrm{v}_{4}$ in the following sections. The Hamilton operators $\stackrel{+}{\boldsymbol{H}}_{4}$ and $\overline{\boldsymbol{H}}_{4}$ [36, Def. 2.1.6] satisfy $\mathrm{v}_{4}\left(\boldsymbol{h} \boldsymbol{h}^{\prime}\right)=\stackrel{+}{\boldsymbol{H}}_{4}(\boldsymbol{h}) \mathrm{v}_{4}\left(\boldsymbol{h}^{\prime}\right)=\overline{\boldsymbol{H}}_{4}\left(\boldsymbol{h}^{\prime}\right) \mathrm{v}_{4}(\boldsymbol{h})$ for $\boldsymbol{h}, \boldsymbol{h}^{\prime} \in \mathbb{H} . \boldsymbol{C}_{4}=\operatorname{diag}(1,-1,-1,-1)$ satisfies $\mathrm{v}_{4}\left(\boldsymbol{h}^{*}\right)=$ $\boldsymbol{C}_{4} \mathrm{v}_{4}(\boldsymbol{h})$ for $\boldsymbol{h} \in \mathbb{H}$. Furthermore, $\overline{\boldsymbol{S}}$ [31, Eq. (3)] has the following properties $\mathrm{v}_{4}\left(\boldsymbol{h} \times \boldsymbol{h}^{\prime}\right)=\overline{\boldsymbol{S}}(\boldsymbol{h}) \mathrm{v}_{4}\left(\boldsymbol{h}^{\prime}\right)=$ $\overline{\boldsymbol{S}}\left(\boldsymbol{h}^{\prime}\right)^{T} \mathrm{v}_{4}(\boldsymbol{h})$ for $\boldsymbol{h}, \boldsymbol{h}^{\prime} \in \mathbb{H}$.

\section{B. Constrained optimization algorithm}

The end effector's tip of two robots can be controlled by a centralized kinematic control strategy [13]. In this work, we only need to control the translation of the instruments' tips because of their symmetry with respect to their shafts. If rotational control is also required, the objective function in [13] can be used.

Let $\boldsymbol{t}_{\mathrm{R} i}, \boldsymbol{t}_{\mathrm{R} i, d} \in \mathbb{H}_{p}$ be the translation and the desired translation of the end effector of each $i$-th robot, with $i \in$ $\{1,2\}$. Given $\boldsymbol{q}=\left[\begin{array}{ll}\boldsymbol{q}_{\mathrm{R} 1}^{T} & \boldsymbol{q}_{\mathrm{R} 2}^{T}\end{array}\right]^{T}$, the desired control signal, $\boldsymbol{u}=\left[\begin{array}{ll}\boldsymbol{u}_{R 1}^{T} & \boldsymbol{u}_{R 2}^{T}\end{array}\right]^{T}$, is obtained as

$$
\begin{aligned}
\boldsymbol{u} & \underset{\dot{\boldsymbol{q}}}{\operatorname{argmin}} \beta\left(f_{t, 1}+f_{\lambda, 1}\right)+(1-\beta)\left(f_{t, 2}+f_{\lambda, 2}\right) \\
& \text { subject to } \boldsymbol{W} \dot{\boldsymbol{q}} \preceq \boldsymbol{w},
\end{aligned}
$$

in which $f_{t, i} \triangleq\left\|\boldsymbol{J}_{t_{i}} \dot{\boldsymbol{q}}_{\mathrm{R} i}+\eta \mathrm{v}_{4}\left(\tilde{\boldsymbol{t}}_{i}\right)\right\|_{2}^{2}$ and $f_{\lambda, i} \triangleq \lambda\left\|\dot{\boldsymbol{q}}_{\mathrm{R} i}\right\|_{2}^{2}$ are the cost functions related to the end-effector translation and joint velocities of the $i$-th robot. Furthermore, each robot has a translation Jacobian $\boldsymbol{J}_{t_{i}} \in \mathbb{R}^{4 \times n_{i}}$ [31, Eq. (6)] that satisfies $\mathrm{v}_{4}\left(\dot{\boldsymbol{t}}_{\mathrm{R} i}\right)=\boldsymbol{J}_{t_{i}} \dot{\boldsymbol{q}}_{\mathrm{R} i}$ and a translation error $\tilde{\boldsymbol{t}}_{i} \triangleq \tilde{\boldsymbol{t}}_{i}\left(\boldsymbol{q}_{\mathrm{R} i}\right)=$ $\boldsymbol{t}_{\mathrm{R} i}-\boldsymbol{t}_{\mathrm{R} i, d}$. In addition, $\eta \in(0, \infty) \subset \mathbb{R}$ is a tunable gain, $\lambda \in[0, \infty) \subset \mathbb{R}$ is a damping factor, and $\beta \in[0,1] \subset \mathbb{R}$ is a weight that defines a "soft" priority between the two robots. A "soft" priority means that there is a relative level of importance between robots, defined by $\beta$, allowing for a good level of customization instead of a strict hierarchical scheme.

The $r$ inequality constraints $\boldsymbol{W} \dot{\boldsymbol{q}} \preceq \boldsymbol{w}$, in which $\boldsymbol{W} \triangleq$ $\boldsymbol{W}(\boldsymbol{q}) \in \mathbb{R}^{r \times\left(n_{1}+n_{2}\right)}, \boldsymbol{w} \triangleq \boldsymbol{w}(\boldsymbol{q}) \in \mathbb{R}^{r}$ are used to generate 
active constraints using the VFI method [31]. Problem (1) in general does not have an analytical solution and a numerical solver must be used. The Lyapunov stability of such approaches has been proven in [37], as long as the objective function is convex and $\boldsymbol{u}=0$ is in the feasible set.

\section{Vector-field-inequalities method}

The vector-field-inequalities (VFI) method [31] is used to map task-space constraints into configuration-space constraints. The VFI method has been shown to be particularly useful for dynamic-active constraints [38, Section III].

The VFI method relies on obtaining functions $d \triangleq d(\boldsymbol{q}, t) \in$ $\mathbb{R}$ that represent the signed distance between two geometric primitives. The time-derivative of the distance is

$$
\dot{d}=\underbrace{\frac{\partial(d(\boldsymbol{q}, t))}{\partial \boldsymbol{q}}}_{\boldsymbol{J}_{d}} \dot{\boldsymbol{q}}+\zeta(t),
$$

where $\boldsymbol{J}_{d} \in \mathbb{R}^{1 \times n}$ is the distance Jacobian and $\zeta(t)=\dot{d}-\boldsymbol{J}_{d} \dot{\boldsymbol{q}}$ is the residual, that contains the distance dynamics unrelated to $\dot{\boldsymbol{q}}$. Moreover, let there be a safe distance $d_{\text {safe }} \triangleq d_{\text {safe }}(t) \in$ $[0, \infty)$ and an error $\tilde{d} \triangleq d_{\text {safe }}-d$ to generate safe zones or $\tilde{d} \triangleq \tilde{d}(\boldsymbol{q}, t)=d-d_{\text {safe }}$ to generate restricted zones.

With these definitions, and given $\eta_{d} \in[0, \infty)$, the signed distance dynamics is constrained by $\tilde{\tilde{d}} \geq-\eta_{d} \tilde{d}$ in both cases, which actively filters the robot motion only in the direction approaching the boundary between the primitives so that the primitives do not collide.

The following constraint is used to generate safe zones, such as the entry-point constraint,

$$
\boldsymbol{J}_{d} \dot{\boldsymbol{q}} \leq \eta_{d} \tilde{d}-\zeta_{\text {safe }}(t),
$$

for $\zeta_{\text {safe }}(t) \triangleq \zeta(t)-\dot{d}_{\text {safe }}$. Alternatively, restricted zones, such as constraints to prevent collisions, are generated by

$$
-\boldsymbol{J}_{d} \dot{\boldsymbol{q}} \leq \eta_{d} \tilde{d}+\zeta_{\text {safe }}(t) .
$$

\section{OVERVIEW OF THE PROPOSED CONTROL STRATEGY}

Our shadow-based autonomous positioning strategy is divided into three steps, as described in Fig. 3: planar positioning, overlap prevention, and vertical positioning. Fig. 4 illustrates the planar and vertical movements of the surgical instrument.

In the planar positioning step described in Section $\mathrm{V}$, the tip of the instrument is moved parallel to the image plane to a safe point above the target region in the retina. The second step, overlap prevention, is described in SectionVI and ensures that the instrument's tip and its shadow do not overlap before the third step begins. Finally, in the vertical positioning step described in Section VII, the instrument is moved vertically to approach the target point in the retina.

\section{PlanAR POSITIONING}

In the planar positioning step, the controller moves the

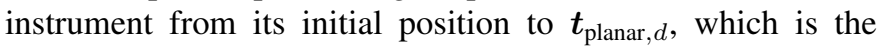
projection of the target position in the retina, $t_{\mathrm{R} 1 \text {, retina }}$, on the plane $\underline{\boldsymbol{\pi}}_{\text {planar }}$, as described in Fig. 4 . Note that the plane $\underline{\boldsymbol{\pi}}_{\text {planar }}$ is parallel to the image plane. To do so, we use the following control law

$$
\begin{gathered}
\boldsymbol{u} \in \underset{\dot{\boldsymbol{q}}}{\operatorname{argmin}} \beta\left(f_{t, 1}+f_{\lambda, 1}\right)+(1-\beta)\left(f_{t, 2}+f_{\lambda, 2}\right) \\
\text { subject to }\left[\begin{array}{c}
\boldsymbol{W}_{\text {vitreo }} \\
\boldsymbol{W}_{\text {shadow }}
\end{array}\right] \dot{\boldsymbol{q}} \preceq\left[\begin{array}{c}
\boldsymbol{w}_{\text {vitreo }} \\
\boldsymbol{w}_{\text {shadow }}
\end{array}\right]
\end{gathered}
$$

with $\boldsymbol{t}_{\mathrm{R} 1, d}=t_{d, \text { plannar }}, \boldsymbol{t}_{\mathrm{R} 2, d}=\mathbf{0}, \beta=0.99, \eta=140$, and $\lambda=0.001$. This causes the light guide to autonomously move in order to keep the constraints while "softly" prioritizing the instrument motion. The inequality constraints simultaneously enforce the vitreoretinal task constraints and shadow-based autonomous positioning constraints using VFIs. The same constraints are used by all steps and are described in detail in Section VIII and Section IX.

This step converges successfully when the error norm goes below a predefined threshold, i.e., $\left\|\tilde{\boldsymbol{t}}_{1}\right\| \leq 0.1 \mathrm{~mm}$, or fails if the error stops decreasing without error convergence within the allowed threshold.

\section{OVERLAP PREVENTION}

After the planar motion finishes successfully, the proposed algorithm moves on to the overlap prevention step. Preventing the overlap between the instrument and its shadow can be achieved by moving the tip of the light guide as far as possible from the plane $\pi_{\mathrm{OP}}$, which contains the $z$-axis of the world frame and the shaft of the instrument as described in Fig. 5, while keeping the tip of the instrument still. To enact the desired motion, we use

$$
\begin{aligned}
\boldsymbol{u} \in \underset{\dot{\boldsymbol{q}}}{\operatorname{argmin}} \| & \boldsymbol{J}_{\mathrm{OP}} \dot{\boldsymbol{q}}-\dot{d}_{\mathrm{OP}, d} \|_{2}^{2}+f_{\lambda, 1}+f_{\lambda, 2} \\
\text { subject to } & {\left[\begin{array}{c}
\boldsymbol{W}_{\text {vitreo }} \\
\boldsymbol{W}_{\text {shadow }}
\end{array}\right] \dot{\boldsymbol{q}} \preceq\left[\begin{array}{c}
\boldsymbol{w}_{\text {vitreo }} \\
\boldsymbol{w}_{\text {shadow }}
\end{array}\right], } \\
\boldsymbol{J}_{t_{1}} & \left.\boldsymbol{O}_{4 \times n_{2}}\right] \dot{\boldsymbol{q}}=\boldsymbol{O}_{4 \times 1}
\end{aligned}
$$

where $d_{\mathrm{OP}}\left(\boldsymbol{q}_{\mathrm{R} 1}, \boldsymbol{q}_{\mathrm{R} 2}\right) \triangleq d_{\mathrm{OP}} \in \mathbb{R}$ is the signed distance between the tip of the light guide and the plane $\pi_{\mathrm{OP}}$ and $\lambda=0.001$. We autonomously control the light guide and try to increase the signed distance $d_{\mathrm{OP}}$ at a constant rate $\dot{d}_{\mathrm{OP}, d}=0.01 \mathrm{~mm} / \mathrm{s}$, which was determined in pilot experiments. The Jacobian $\boldsymbol{J}_{\mathrm{OP}} \in \mathbb{R}^{1 \times\left(n_{1}+n_{2}\right)}$ satisfies $\dot{d}_{\mathrm{OP}}=\boldsymbol{J}_{\mathrm{OP}} \dot{\boldsymbol{q}}$. The distance function, $d_{\mathrm{OP}}$, and Jacobian, $\boldsymbol{J}_{\mathrm{OP}}$, are described in detail in Section VI-A and Section VI-B, respectively.

Given that it is not possible to know beforehand how far the light guide can move further from $\pi_{\mathrm{OP}}$, the overlap prevention step runs until the distance between the shadow's tip and the surgical instrument's shaft, $d_{\text {shaft }}$ gets larger than the predefined threshold, $k_{\text {overlap}}$, in the microscopic view. If the distance $d_{\text {shaft }}$ stops increasing but it is not over $k_{\text {overlap }}$, the controller does not advance to the next step.

\section{A. The signed distance function $d_{O P}$}

As shown in Fig. 5, let the world reference-frame $\mathcal{F}_{\mathrm{W}}$ be in the retina plane with its $z$-axis pointing upwards, and define

$$
\boldsymbol{t}_{\mathrm{R} 1}^{\mathrm{R} 2}\left(\boldsymbol{q}_{\mathrm{R} 1}, \boldsymbol{q}_{\mathrm{R} 2}\right) \triangleq \boldsymbol{t}_{\mathrm{R} 1}^{\mathrm{R} 2}=\boldsymbol{t}_{\mathrm{R} 1}-\boldsymbol{t}_{\mathrm{R} 2} .
$$



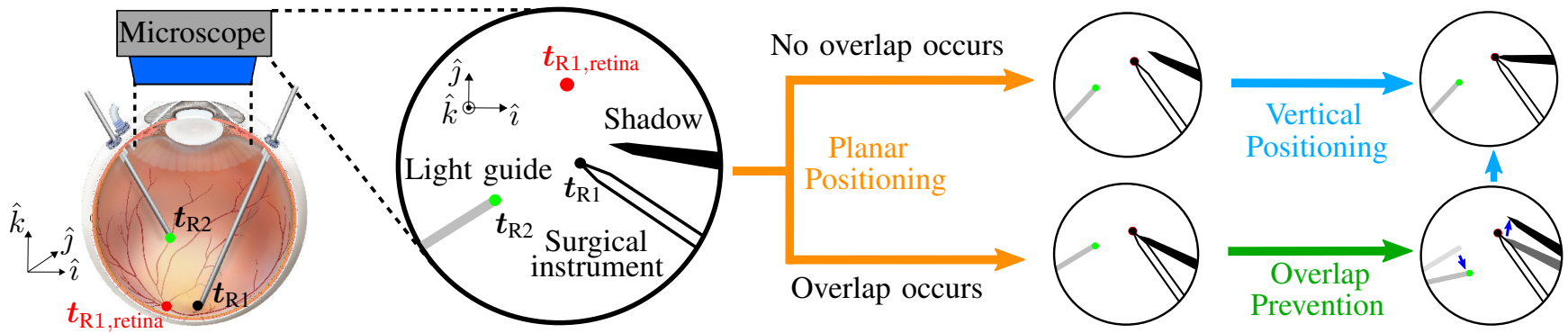

Fig. 3. The entire process of the proposed shadow-based autonomous positioning of the surgical instrument's tip to the retina surface. The proposed method completes positioning in three steps at most. If the overlap of the surgical instrument's shaft and its shadow in the microscopic view occurs after the planar positioning step, the controller removes this overlap in the overlap prevention step to use the shadow in the vertical positioning step.

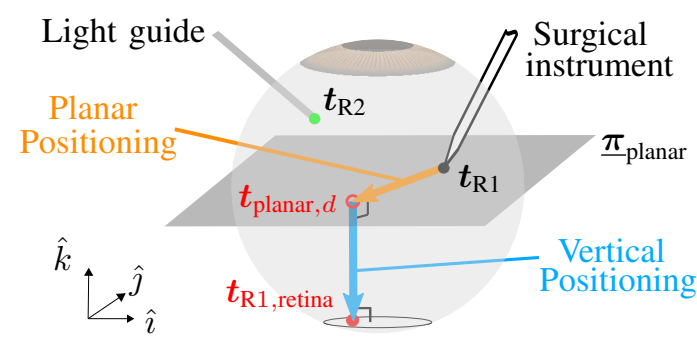

Fig. 4. The planar and vertical movements of the tip of the surgical instrument. In the planar positioning step, the tip is moved parallel to the image plane to a safe point, $\boldsymbol{t}_{\mathrm{planar}, d}$, above the target, $\boldsymbol{t}_{t_{\mathrm{R} 1} \text {, retina }}$.

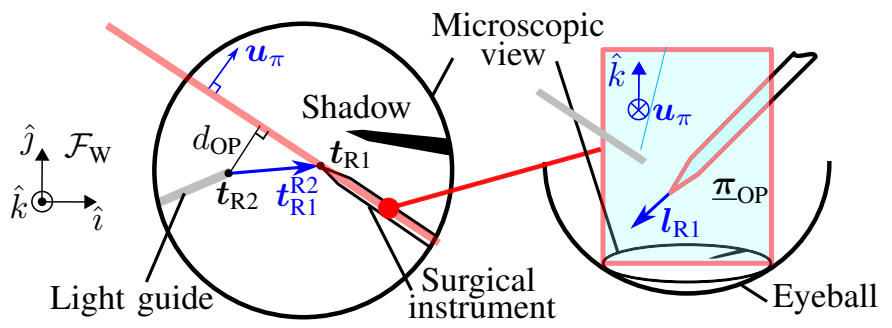

Fig. 5. The plane, $\underline{\pi}_{\mathrm{OP}}$, used to prevent the overlap of the surgical instrument's shaft and its shadow. When the light guide's tip is not on $\underline{\boldsymbol{\pi}}_{\mathrm{OP}}$, the overlap does not happen.

Let the rotation of the tip of the surgical instrument be the unit quaternion $\boldsymbol{r}_{\mathrm{R} 1}\left(\boldsymbol{q}_{\mathrm{R} 1}\right) \triangleq \boldsymbol{r}_{\mathrm{R} 1} \in \mathbb{S}^{3}$. In addition, let the unit pure quaternion $\boldsymbol{u}_{\pi} \in \mathbb{H}_{p} \cap \mathbb{S}^{3}$ represent the unit normal of the plane. Moreover, let $\boldsymbol{l}_{\mathrm{R} 1}\left(\boldsymbol{q}_{\mathrm{R} 1}\right) \triangleq \boldsymbol{l}_{\mathrm{R} 1} \in \mathbb{H}_{p} \cap \mathbb{S}^{3}$ be the direction of the $z$-axis of the surgical instrument (pointing outwards, collinear with the shaft of the surgical instrument); that is, $\boldsymbol{l}_{\mathrm{R} 1}=\boldsymbol{r}_{\mathrm{R} 1} \hat{k} \boldsymbol{r}_{\mathrm{R} 1}^{*}$. Then, since the unit plane normal $\boldsymbol{u}_{\pi}$ is orthogonal to both the $z$-axis of the frame $\mathcal{F}_{\mathrm{W}}, \hat{k}$, and the direction of the $z$-axis of the surgical instrument, $l_{\mathrm{R} 1}$, we get ${ }^{2}$

$$
\boldsymbol{n}_{\pi}=\boldsymbol{l}_{\mathrm{R} 1} \times \hat{k}, \boldsymbol{u}_{\pi}=\frac{\boldsymbol{n}_{\pi}}{\left\|\boldsymbol{n}_{\pi}\right\|} .
$$

Then, from (6) and (7) and using the inner product, we can get the signed distance $d_{\mathrm{OP}}$ as follows

$$
d_{\mathrm{OP}}=\left\langle\boldsymbol{u}_{\pi}, \boldsymbol{t}_{\mathrm{R} 1}^{\mathrm{R}}\right\rangle .
$$

\footnotetext{
${ }^{2}$ The unit plane normal $\boldsymbol{u}_{\pi}$ is singular when $\boldsymbol{l}_{\mathrm{R} 1} \times \hat{k}=0$, that is, when the surgical instrument's shaft is parallel to the $z$-axis of the frame $\mathcal{F}_{\mathrm{W}}$. However, this situation never happens inside the workspace, and this movement is prevented by the constraint $\mathrm{C}_{\mathrm{m}}$.
}

\section{B. The corresponding Jacobian $\boldsymbol{J}_{O P}$}

The corresponding Jacobian $\boldsymbol{J}_{\mathrm{OP}}$ relates the joint velocities $\dot{\boldsymbol{q}}$ to the time derivatives of the signed distance $d_{\mathrm{OP}}$. From (8), the time derivative of the signed distance is

$$
\dot{d}_{\mathrm{OP}}=\left\langle\boldsymbol{u}_{\pi}, \dot{\boldsymbol{t}}_{\mathrm{R} 1}^{\mathrm{R} 2}\right\rangle+\left\langle\boldsymbol{t}_{\mathrm{R} 1}^{\mathrm{R} 2}, \dot{\boldsymbol{u}}_{\pi}\right\rangle .
$$

Then, we have to find $\dot{\boldsymbol{t}}_{\mathrm{R} 1}^{\mathrm{R} 2}$ and $\dot{\boldsymbol{u}}_{\pi}$ with respect to $\dot{\boldsymbol{q}}$. From (6), we have

$$
\mathrm{v}_{4}\left(\dot{\boldsymbol{t}}_{\mathrm{R} 1}^{\mathrm{R} 2}\right)=\mathrm{v}_{4}\left(\dot{\boldsymbol{t}}_{\mathrm{R} 1}-\dot{\boldsymbol{t}}_{\mathrm{R} 2}\right)=\underbrace{\left[\begin{array}{ll}
\boldsymbol{J}_{t_{1}}-\boldsymbol{J}_{t_{2}}
\end{array}\right]}_{\boldsymbol{J}_{\mathrm{R} 1}^{\mathrm{R}}} \dot{\boldsymbol{q}} .
$$

From (7) and using the property [39, Eq. (3)], we have

$$
\mathrm{v}_{4}\left(\dot{\boldsymbol{u}}_{\pi}\right)=\underbrace{\left(\frac{\boldsymbol{I}_{4 \times 4}}{\left\|\boldsymbol{n}_{\pi}\right\|}-\frac{\mathrm{v}_{4}\left(\boldsymbol{n}_{\pi}\right) \mathrm{v}_{4}\left(\boldsymbol{n}_{\pi}\right)^{T}}{\left\|\boldsymbol{n}_{\pi}\right\|^{3}}\right)}_{\boldsymbol{u}_{1}} \mathrm{v}_{4}\left(\dot{\boldsymbol{n}}_{\pi}\right) .
$$

Moreover,

$$
\begin{gathered}
\mathrm{v}_{4}\left(\dot{\boldsymbol{n}}_{\pi}\right)=\mathrm{v}_{4}\left(\dot{\boldsymbol{l}}_{\mathrm{R} 1} \times \hat{k}\right) \\
=\underbrace{\overline{\boldsymbol{S}}(\hat{k})^{T}\left(\overline{\boldsymbol{H}}_{4}\left(\hat{k} \boldsymbol{r}_{\mathrm{R} 1}^{*}\right)+\stackrel{+}{\boldsymbol{H}}_{4}\left(\boldsymbol{r}_{\mathrm{R} 1} \hat{k}\right) \boldsymbol{C}_{4}\right) \boldsymbol{J}_{r_{1}}}_{\boldsymbol{J}_{n_{\pi}}} \dot{\boldsymbol{q}}_{\mathrm{R} 1},
\end{gathered}
$$

where $\boldsymbol{J}_{r_{1}} \in \mathbb{R}^{4 \times n_{1}}$ is the rotation Jacobian [31, Eq. (7)]. Substituting (12) into (11), we find

$$
\mathrm{v}_{4}\left(\dot{u}_{\pi}\right)=\underbrace{\left[\begin{array}{ll}
u_{1} J_{n_{\pi}} & \boldsymbol{O}_{4 \times 6}
\end{array}\right]}_{J_{u_{\pi}}} \dot{\boldsymbol{q}} .
$$

Finally, substituting (10) and (13) into (9), we get

$$
\dot{d}_{\mathrm{OP}}=\underbrace{\left(\mathrm{v}_{4}\left(\boldsymbol{u}_{\pi}\right)^{T} \boldsymbol{J}_{\mathrm{R} 1}^{\mathrm{R} 2}+\mathrm{v}_{4}\left(\boldsymbol{t}_{\mathrm{R} 1}^{\mathrm{R} 2}\right)^{T} \boldsymbol{J}_{\boldsymbol{u}_{\pi}}\right)}_{\boldsymbol{J}_{\mathrm{OP}}} \dot{\boldsymbol{q}} .
$$

\section{VERTICAL POSITIONING}

After a successful overlap-prevention step, the controller moves on to the vertical positioning step. The main goal is to get the tip of the surgical instrument onto the retina precisely, so we prioritize the motion of the instrument over that of the light guide using a quadratic programming $(\mathrm{QP})$-based taskpriority framework [40]. 
To enact the desired motion, we use two cascaded QP optimizations

$$
\begin{aligned}
& \boldsymbol{u} \in \underset{\dot{\boldsymbol{q}}}{\operatorname{argmin}}\left\|\boldsymbol{J}_{\mathrm{OP}} \dot{\boldsymbol{q}}-\dot{d}_{\text {Desired }}\right\|_{2}^{2}+f_{\lambda, 1}+f_{\lambda, 2} \\
& \text { subject to } {\left[\begin{array}{c}
\boldsymbol{W}_{\text {vitreo }} \\
\boldsymbol{W}_{\text {shadow }}
\end{array}\right] \dot{\boldsymbol{q}} \preceq\left[\begin{array}{c}
\boldsymbol{w}_{\text {vitreo }} \\
\boldsymbol{w}_{\text {shadow }}
\end{array}\right] } \\
&\left.\begin{array}{lll}
\boldsymbol{J}_{t_{1}} & \boldsymbol{O}_{4 \times n_{2}}
\end{array}\right] \dot{\boldsymbol{q}}=\left[\begin{array}{lll}
\boldsymbol{J}_{t_{1}} & \boldsymbol{O}_{4 \times n_{2}}
\end{array}\right] \boldsymbol{u}^{\prime}
\end{aligned}
$$

where

$$
\begin{aligned}
\boldsymbol{u}^{\prime} \in \underset{\dot{\boldsymbol{q}}}{\operatorname{argmin}}\left\|\boldsymbol{J}_{t_{1}} \dot{\boldsymbol{q}}_{\mathrm{R} 1}+\eta \mathrm{v}_{4}\left(\tilde{\boldsymbol{t}}_{1}\right)\right\|_{2}^{2}+f_{\lambda, 1}+f_{\lambda, 2} \\
\text { subject to }\left[\begin{array}{c}
\boldsymbol{W}_{\text {vitreo }} \\
\boldsymbol{W}_{\text {shadow }}
\end{array}\right] \dot{\boldsymbol{q}} \preceq\left[\begin{array}{c}
\boldsymbol{w}_{\text {vitreo }} \\
\boldsymbol{w}_{\text {shadow }}
\end{array}\right],
\end{aligned}
$$

with $t_{\mathrm{R} 1, d}=t_{\mathrm{R} 1 \text {,retina }}, \eta=150$, and $\lambda=0.0005$. The parameters of (15) are the same as those of (5). This causes the tip of the instrument to move toward the point in the retina, while trying, as much as possible, to increase the distance between the tip of the light guide and $\pi_{\mathrm{OP}}$.

This step converges when the distance between the surgical instrument's tip and the shadow's tip, $d_{\text {tip }}$, gets smaller than the predefined threshold, $k_{\text {vertical }}$, in the microscopic view.

\section{VITREORETINAL TASK CONSTRAINTS}

(a)

$\mathrm{C}_{\mathrm{R}}$ Line-to-point

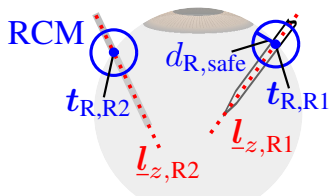

(b) $\quad \mathrm{C}_{\mathrm{r}}$ Point-to-point

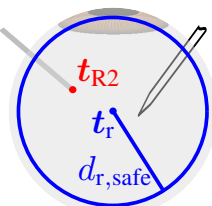

(e) $\mathrm{C}_{\mathrm{t}}$

(d) $\mathrm{C}_{\mathrm{tr}}$ Point-to-point

Trocar

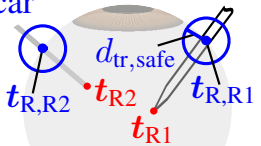

Point-to-point (c) $\mathrm{C}_{\mathrm{s}}$ Point-to-line

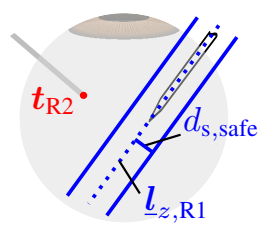

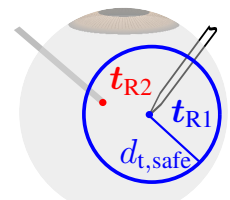

This section describes how to enforce the vitreoretinal task constraints introduced in Section II-C using the VFI method and the Jacobians and distance functions described in [31, Section IV]. Fig. 6 and Fig. 7 illustrate the pairs of geometrical primitives and the type of VFI we use for each pair.

For $\mathrm{C}_{R}$ and $\mathrm{C}_{r}$, based on (2), we use

$$
\underbrace{\left[\begin{array}{cc}
\boldsymbol{J}_{\mathrm{R}, 1} & \boldsymbol{O}_{1 \times n_{2}} \\
\boldsymbol{O}_{1 \times n_{1}} & \boldsymbol{J}_{\mathrm{R}, 2} \\
\boldsymbol{O}_{1 \times n_{1}} & \boldsymbol{J}_{\mathrm{r}, 2}
\end{array}\right]}_{\boldsymbol{W}_{\mathrm{R}, \mathrm{r}}} \dot{\boldsymbol{q}} \preceq \underbrace{\left[\begin{array}{c}
\eta_{\mathrm{R}}\left(D_{\mathrm{R}, \text { safe }}-D_{\mathrm{R}, 1}\right) \\
\eta_{\mathrm{R}}\left(D_{\mathrm{R}, \text { safe }}-D_{\mathrm{R}, 2}\right) \\
\eta_{\mathrm{r}}\left(D_{\mathrm{r}, \text { safe }}-D_{\mathrm{r}, 2}\right)
\end{array}\right]}_{\boldsymbol{w}_{\mathrm{R}, \mathrm{r}}},
$$

where $D_{\mathrm{R}, i}$ and $\boldsymbol{J}_{\mathrm{R}, i}$ are the line-to-point squared distances and Jacobians [31, Eq. (33), (34)] between the Plücker lines collinear with the shafts, $\underline{\boldsymbol{l}}_{z, i}$, and the RCM points, $\boldsymbol{t}_{\mathrm{R}, \mathrm{R} i}$. Moreover, $D_{\mathrm{r}, 2}$ and $\boldsymbol{J}_{\mathrm{r}, 2}$ are the point-to-point squared distance and Jacobian [31, Eq. (21), (22)] between the light guide's tip, $t_{\mathrm{R} 2}$, and the center point of the eyeball, $\boldsymbol{t}_{\mathrm{r}}$. Furthermore, $\eta_{\mathrm{R}}=\eta_{\mathrm{r}}=0.01$, and $D_{\mathrm{R}, \text { safe }}=\left(d_{\mathrm{R} \text {,safe }}\right)^{2}=(0.5 \mathrm{~mm})^{2}$, and $D_{\mathrm{r}, \text { safe }}=\left(d_{\mathrm{r}, \text { safe }}\right)^{2}=(10 \mathrm{~mm})^{2}$.

To enforce $\mathrm{C}_{\mathrm{s}}{ }^{3}, \mathrm{C}_{\mathrm{tr}}$, and $\mathrm{C}_{\mathrm{m}}$, based on (3), we use

$$
\underbrace{\left[\begin{array}{cc}
\boldsymbol{O}_{1 \times n_{1}} & -\boldsymbol{J}_{\mathrm{s}, 2} \\
-\boldsymbol{J}_{\mathrm{tr}, 1} & \boldsymbol{O}_{1 \times n_{2}} \\
\boldsymbol{O}_{1 \times n_{1}} & -\boldsymbol{J}_{\mathrm{tr}, 2} \\
-\boldsymbol{J}_{\mathrm{m}, 1} & \boldsymbol{O}_{1 \times n_{2}} \\
\boldsymbol{O}_{1 \times n_{1}} & -\boldsymbol{J}_{\mathrm{m}, 2}
\end{array}\right]}_{\boldsymbol{W}_{\mathrm{s}, \mathrm{tr}, \mathrm{m}}} \dot{\boldsymbol{q}} \preceq \underbrace{\left[\begin{array}{c}
\eta_{\mathrm{s}}\left(D_{\mathrm{s}, 2}-D_{\mathrm{s}, \mathrm{safe}}\right) \\
\eta_{\mathrm{tr}}\left(D_{\mathrm{tr}, 1}-D_{\mathrm{tr}, \mathrm{safe}}\right) \\
\eta_{\mathrm{tr}}\left(D_{\mathrm{tr}, 2}-D_{\mathrm{tr}, \mathrm{safe}}\right) \\
\eta_{\mathrm{m}}\left(D_{\mathrm{m}, 1}-D_{\mathrm{m}, \mathrm{safe}}\right) \\
\eta_{\mathrm{m}}\left(D_{\mathrm{m}, 2}-D_{\mathrm{m}, \mathrm{safe}}\right)
\end{array}\right]}_{\boldsymbol{w}_{\mathrm{s}, \mathrm{tr}, \mathrm{m}}},
$$

where $D_{\mathrm{s}, 2}$ and $\boldsymbol{J}_{\mathrm{s}, 2}$ are the point-to-line squared distance and Jacobian [31, Eq. (29), (32)] between $\boldsymbol{t}_{\mathrm{R} 2}$ and $\underline{l}_{z, 1}$, and $D_{\mathrm{tr}, i}$ and $\boldsymbol{J}_{\mathrm{tr}, i}$ are the point-to-point squared distances and Jacobians between $\boldsymbol{t}_{\mathrm{Ri}}$ and the RCM points, $\boldsymbol{t}_{\mathrm{R}, \mathrm{R} i}$. Moreover, $D_{\mathrm{m}, i}$ and $\boldsymbol{J}_{\mathrm{m}, i}$ are the point-to-line squared distances and Jacobians between the $n_{i}$-th joints of the robots, $\boldsymbol{t}_{n_{i}, \mathrm{R} i}$, which are most likely to collide with the microscope, and the Plücker line collinear with the microscope direction, $\underline{\boldsymbol{l}}_{z, \mathrm{~m}}$. Furthermore, $\eta_{\mathrm{s}}=\eta_{\mathrm{m}}=1, \eta_{\mathrm{tr}}=0.01, D_{\mathrm{s}, \mathrm{safe}}=\left(d_{\mathrm{s}, \mathrm{safe}}\right)^{2}=(0.5 \mathrm{~mm})^{2}$, $D_{\mathrm{tr}, \mathrm{safe}}=\left(d_{\mathrm{tr}, \mathrm{safe}}\right)^{2}=(5 \mathrm{~mm})^{2}$, and $D_{\mathrm{m}, \mathrm{safe}}=(6 \mathrm{~cm})^{2}$.

For $\mathrm{C}_{\mathrm{ro}}$, we constrain the $l_{k}-$ th, $l_{k} \in\left\{2, \cdots, n_{i}\right\}$, joint of

Fig. 6. The geometrical primitives and types of VFIs used to generate each task constraint related to the eye. (a), (b), (c), and (d) show how the vitreoretinal task constraints $\mathrm{C}_{x}(x=\mathrm{R}, \mathrm{r}, \mathrm{s}, \mathrm{tr})$ are generated. (e) shows how the shadow-based autonomous positioning constraint $\mathrm{C}_{t}$ is generated. Note that the trocar points described in (d) correspond with the RCM points described in (a).

(a) $\mathrm{C}_{\mathrm{m}}$ Point-to-line

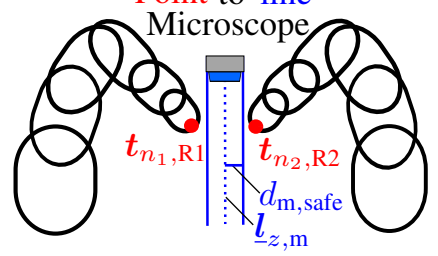

(b)

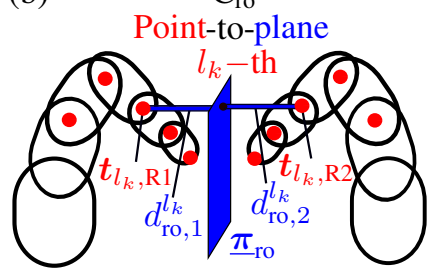

Fig. 7. The geometrical primitives and types of VFIs used to generate each task constraint related to the robots. (a) and (b) show how the vitreoretinal task constraints $C_{m}$ and $C_{r o}$ are generated, respectively. the robot $\mathrm{R}_{i}, \boldsymbol{t}_{l_{k}, \mathrm{R} i}$, to have a given distance from the plane $\underline{\boldsymbol{\pi}}_{\mathrm{ro}}$ described in Fig. 7-(b). Let the signed distances and Jacobians [31, Eq. (57), (59)] between $\boldsymbol{t}_{l_{k}, \mathrm{R} i}$ and $\underline{\boldsymbol{\pi}}_{\mathrm{ro}}$ be $d_{\mathrm{ro}, i}^{l_{k}} \in \mathbb{R}$ and $\boldsymbol{J}_{x, i}^{l_{k}} \in \mathbb{R}^{1 \times n_{i}}$, respectively. We can use

$$
\underbrace{\left[\begin{array}{ll}
-\boldsymbol{J}_{\mathrm{ro}, 1} & \boldsymbol{O}_{1 \times n_{2}} \\
\boldsymbol{O}_{1 \times n_{1}} & -\boldsymbol{J}_{\mathrm{ro}, 2}
\end{array}\right]}_{\boldsymbol{W}_{\mathrm{ro}}} \dot{\boldsymbol{q}} \preceq \underbrace{\eta_{\mathrm{ro}}\left[\begin{array}{c}
d_{\mathrm{ro}, 1} \\
d_{\mathrm{ro}, 2}
\end{array}\right]}_{\boldsymbol{w}_{\mathrm{ro}}},
$$

\footnotetext{
${ }^{3}$ The possible collision between the light guide and the surgical instrument is only the collision between the light guide's tip and the surgical instrument's shaft. This is because the light guide's tip is always above the surgical instrument's shaft to illuminate the surgical instrument's tip. This is ensured with the constraint $C_{2}$ described in Section II-D. Therefore, we use a pointto-line VFI method for the constraint $\mathrm{C}_{\mathrm{S}}$. Nonetheless, in other scenarios, a shaft-to-shaft collision avoidance strategy could be used as implemented in [31].
} 
where $\eta_{\text {ro }}=1$ and

$$
\boldsymbol{J}_{\mathrm{ro}, i} \triangleq\left[\begin{array}{cc}
\boldsymbol{J}_{\mathrm{ro}, i}^{2} & \boldsymbol{O}_{1 \times\left(n_{i}-2\right)} \\
\vdots & \\
\boldsymbol{J}_{\mathrm{ro}, i}^{n_{i}-1} & \boldsymbol{O}_{1 \times 1} \\
& \boldsymbol{J}_{\mathrm{ro}, i}^{n_{i}}
\end{array}\right], d_{\mathrm{ro}, i} \triangleq\left[\begin{array}{c}
d_{\mathrm{ro}, i}^{2} \\
\vdots \\
d_{\mathrm{ro}, i}^{n_{i}-1} \\
d_{\mathrm{ro}, i}^{n_{i}}
\end{array}\right] .
$$

The linear inequality constraints can also be used to enforce joint limits $\mathrm{C}_{\mathrm{j}}$ [41]

$$
\underbrace{\left[\begin{array}{ll}
W_{J L, 1} & \boldsymbol{O}_{1 \times n_{2}} \\
\boldsymbol{O}_{1 \times n_{1}} & W_{J L, 2}
\end{array}\right]}_{\boldsymbol{W}_{J L}} \dot{\boldsymbol{q}} \preceq \underbrace{\left[\begin{array}{c}
w_{J L, 1} \\
w_{J L, 2}
\end{array}\right]}_{\boldsymbol{w}_{J l}},
$$

where

$$
W_{J L, i} \triangleq\left[\begin{array}{c}
-\boldsymbol{I}_{n_{i} \times n_{i}} \\
\boldsymbol{I}_{n_{i} \times n_{i}}
\end{array}\right], w_{J L, i} \triangleq\left[\begin{array}{c}
\boldsymbol{q}_{\min , i}-\boldsymbol{q}_{\mathrm{R} i} \\
\boldsymbol{q}_{\max , i}-\boldsymbol{q}_{\mathrm{R} i}
\end{array}\right],
$$

and $\boldsymbol{q}_{\min , i}, \boldsymbol{q}_{\max , i} \in \mathbb{R}^{n_{i}}$ are the lower and upper bounds of the joint values.

In conclusion, the vitreoretinal constraints can be enforced using the following inequality constraint based on (17)-(20)

$$
\underbrace{\left[\begin{array}{c}
\boldsymbol{W}_{\mathrm{R}, \mathrm{r}} \\
\boldsymbol{W}_{\mathrm{s}, \mathrm{tr}, \mathrm{m}} \\
\boldsymbol{W}_{\mathrm{ro}} \\
\boldsymbol{W}_{\text {limit }}
\end{array}\right]}_{\boldsymbol{W}_{\text {vitreo }}} \dot{\boldsymbol{q}} \preceq \underbrace{\left[\begin{array}{c}
\boldsymbol{w}_{\mathrm{R}, \mathrm{r}} \\
\boldsymbol{w}_{\mathrm{s}, \mathrm{tr}, \mathrm{m}} \\
\boldsymbol{w}_{\text {ro }} \\
\boldsymbol{w}_{\text {limit }}
\end{array}\right]}_{\boldsymbol{w}_{\text {vitreo }}} \text {. }
$$

\section{ShadOW-BASED POSITIONING CONSTRAINTS}

This section describes how to enforce the shadow-based autonomous positioning constraints introduced in Section II-D. Firstly, as described in Fig. 6-(e), $C_{t}$ can be generated using the point-to-point Jacobian $\boldsymbol{J}_{\mathrm{t}, 2}$ and the point-to-point squared distance $D_{\mathrm{t}, 2}$ between $\boldsymbol{t}_{\mathrm{R} 2}$ and $\boldsymbol{t}_{\mathrm{R} 1}$ based on (2):

$$
\underbrace{\left[\begin{array}{ll}
\boldsymbol{O}_{1 \times n_{1}} & \boldsymbol{J}_{\mathrm{t}, 2}
\end{array}\right]}_{\boldsymbol{W}_{\mathrm{t}}} \dot{\boldsymbol{q}} \preceq \underbrace{\eta_{\mathrm{t}}\left(D_{\mathrm{t}, \mathrm{safe}}-D_{\mathrm{t}, 2}\right)}_{\boldsymbol{w}_{\mathrm{t}}},
$$

with $\eta_{\mathrm{t}} \triangleq 0.01$ and $D_{\mathrm{t}, \mathrm{safe}}=\left(d_{\mathrm{t}, \mathrm{safe}}\right)^{2} \triangleq(10 \mathrm{~mm})^{2}$.

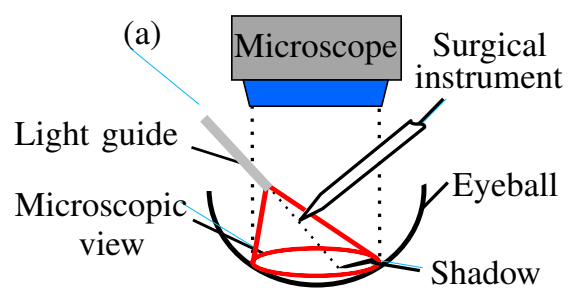

(b)
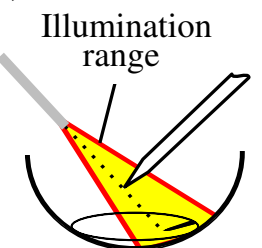

Fig. 8. Conical constraints to enforce $\mathrm{C}_{1}$ and $\mathrm{C}_{2}$ needed to guarantee the visibility of the shadow of the surgical instrument's tip inside the circular microscopic view. (a) In geometrical terms, the tip of the shadow is visible through the microscopic as long as the surgical instrument's tip is inside the cone formed by the light guide's tip and the circular microscopic view. (b) The tip of the surgical instrument is illuminated as long as the surgical instrument's tip is inside the illumination volume of the light guide, which is a cone.

We can generate $\mathrm{C}_{1}$ and $\mathrm{C}_{2}$ by keeping the tip of the surgical instrument inside the two red cones described in Fig. 8: the cone formed by the light guide's tip and the circular microscopic view and the cone which corresponds with the illumination volume of the light guide.
Quiroz-Omana et al. [42] proposed a singularity-free conical constraint based on the VFI for cones whose central axis never changes. In our use case, the direction of the central axis shifts depending on the pose of the light guide, so we propose suitable dynamic conical VFIs that make use of the geometry of the vitreoretinal task.

Let the signed distances between the surgical instrument's tip and the time-dependent boundaries of the proposed conical zones be $\tilde{d}_{\mathrm{C}_{1}} \triangleq d_{\mathrm{C}_{1}}-d_{\mathrm{C}_{1} \text {, safe }} \in \mathbb{R}$ and $\tilde{d}_{\mathrm{C}_{2}} \triangleq d_{\mathrm{C}_{2}}-d_{\mathrm{C}_{2} \text {, safe }} \in \mathbb{R}$ based on the relationships (28) and (62), respectively. Then, based on (3), the constraints can be implemented as the following linear constraints

$$
\underbrace{\left[\begin{array}{c}
-\left(\boldsymbol{J}_{\mathrm{C}_{1}}-\boldsymbol{J}_{\mathrm{C}_{1}, \text { safe }}\right) \\
-\left(\boldsymbol{J}_{\mathrm{C}_{2}}-\boldsymbol{J}_{\mathrm{C}_{2}, \text { safe }}\right) \\
\boldsymbol{W}_{\mathrm{t}}
\end{array}\right]}_{\boldsymbol{W}_{\text {shadow }}} \dot{\boldsymbol{q}} \preceq \underbrace{\left[\begin{array}{c}
\eta_{1}\left(d_{\mathrm{C}_{1}}-d_{\mathrm{C}_{1}, \text { safe }}\right) \\
\eta_{2}\left(d_{\mathrm{C}_{2}}-d_{\mathrm{C}_{2}, \text { safe }}\right) \\
\boldsymbol{w}_{\mathrm{t}}
\end{array}\right]}_{\boldsymbol{w}_{\text {shadow }}},
$$

where $\eta_{1}=\eta_{2}=0.1$. Furthermore, $\boldsymbol{J}_{\mathrm{C}_{1}}-\boldsymbol{J}_{\mathrm{C}_{1} \text {,safe }}, \boldsymbol{J}_{\mathrm{C}_{2}}-$ $\boldsymbol{J}_{\mathrm{C}_{2} \text {, safe }} \in \mathbb{R}^{1 \times n}$ are the corresponding Jacobians that relate the joint velocities to the time derivatives of $\tilde{d}_{\mathrm{C}_{1}}, \tilde{d}_{\mathrm{C}_{2}}$.

\section{A. Distance function and safe distance function for $C_{1}$}

In this section, the goal is to find a distance function and a safe distance function ${ }^{4}$ to enforce $\mathrm{C}_{1}$. The distance function is related with the distance between the center of the microscopic view and the tip of the shadow of the instrument projected on the retina,

$$
d_{\mathrm{C}_{1}}\left(\boldsymbol{q}_{\mathrm{R} 1}, \boldsymbol{q}_{\mathrm{R} 2}\right) \triangleq d_{\mathrm{C}_{1}} \in \mathbb{R}^{+} .
$$

The safe distance function delineates the edge of the microscopic view,

$$
d_{\mathrm{C}_{1}, \text { safe }}\left(\boldsymbol{q}_{\mathrm{R} 1}, \boldsymbol{q}_{\mathrm{R} 2}\right) \triangleq d_{\mathrm{C}_{1}, \mathrm{safe}} \in \mathbb{R}^{+}
$$

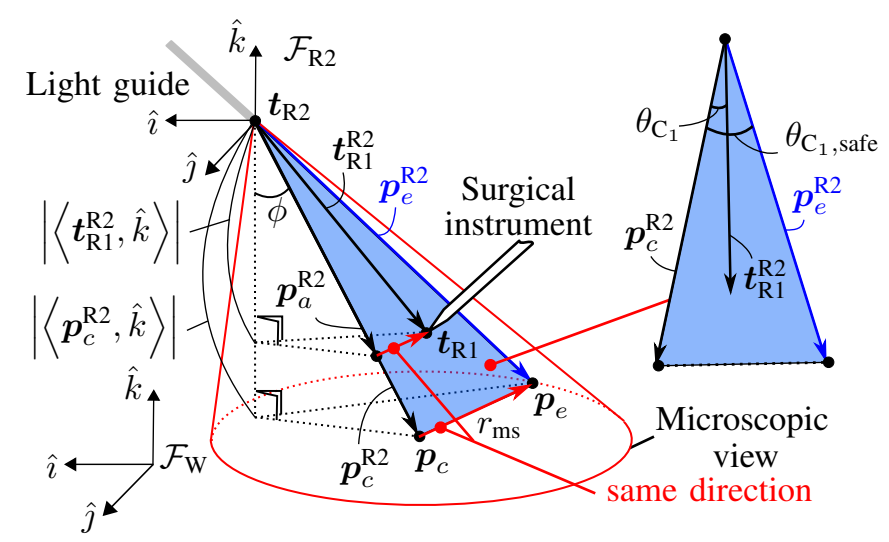

Fig. 9. The relationship between $\theta_{\mathrm{C}_{1}}$ and $\theta_{\mathrm{C}_{1} \text {, safe }}$ used to define the distance

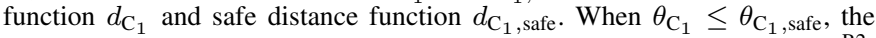
tip of the surgical instrument is kept inside the proposed cone. To find $\boldsymbol{p}_{e}^{\mathrm{R} 2}$, we have to find $\boldsymbol{p}_{e}$, the point in the edge of the microscopic view and on the plane spanned by $\boldsymbol{p}_{c}, \boldsymbol{t}_{\mathrm{R} 1}$, and $\boldsymbol{t}_{\mathrm{R} 2}$.

In Fig. 9, we use the same symbols as in Fig. 5: the world reference-frame, $\mathcal{F}_{\mathrm{W}}$, and the translations of the tips, $t_{\mathrm{R} 1}$ and

\footnotetext{
${ }^{4}$ There are many possible combinations of $d_{\mathrm{C}_{1}}$ and $d_{\mathrm{C}_{1} \text {, safe }}$ that can generate $\mathrm{C}_{1}$. In this work, we discuss one of those ways.
} 
$\boldsymbol{t}_{\mathrm{R} 2}$. In addition, let $\boldsymbol{p}_{c} \in \mathbb{H}_{p}$ represent the center of the circular microscopic view with radius $r_{\mathrm{ms}} \in \mathbb{R}^{+}-\{0\}$. Moreover, let $\boldsymbol{p}_{e}\left(\boldsymbol{q}_{\mathrm{R} 1}, \boldsymbol{q}_{\mathrm{R} 2}\right) \triangleq \boldsymbol{p}_{e} \in \mathbb{H}_{p}$ be the point in the edge of the microscopic view closest to $t_{\mathrm{R} 1}$, hence located on the plane spanned by $\boldsymbol{p}_{c}, \boldsymbol{t}_{\mathrm{R} 1}$ and $\boldsymbol{t}_{\mathrm{R} 2}$.

To simplify the following explanation, let $\mathcal{F}_{\mathrm{R} 2}$ be the reference frame with the same orientation as $\mathcal{F}_{\mathrm{W}}$ and whose origin coincides with $\boldsymbol{t}_{\mathrm{R} 2}$. We can re-write $\boldsymbol{t}_{\mathrm{R} 1}, \boldsymbol{p}_{c}$ and $\boldsymbol{p}_{e}$ with respect to $\mathcal{F}_{\mathrm{R} 2}$ as $(6), \boldsymbol{p}_{c}^{\mathrm{R} 2}\left(\boldsymbol{q}_{\mathrm{R} 2}\right) \triangleq \boldsymbol{p}_{c}^{\mathrm{R} 2}$ and $\boldsymbol{p}_{e}^{\mathrm{R} 2}\left(\boldsymbol{q}_{\mathrm{R} 1}, \boldsymbol{q}_{\mathrm{R} 2}\right) \triangleq \boldsymbol{p}_{e}^{\mathrm{R} 2}$, respectively.

With the above definitions, let $\theta_{\mathrm{C}_{1}}\left(\boldsymbol{q}_{\mathrm{R} 1}, \boldsymbol{q}_{\mathrm{R} 2}\right) \triangleq \theta_{\mathrm{C}_{1}} \in$ $[0, \pi / 2) \subset \mathbb{R}$ be the angle between $\boldsymbol{t}_{\mathrm{R} 1}^{\mathrm{R} 2}$ and $\boldsymbol{p}_{c}^{\mathrm{R} 2}$; and $\theta_{\mathrm{C}_{1}, \text { safe }}\left(\boldsymbol{q}_{\mathrm{R} 1}, \boldsymbol{q}_{\mathrm{R} 2}\right) \triangleq \theta_{\mathrm{C}_{1} \text {, safe }} \in(0, \pi / 2)$ be the angle between $\boldsymbol{p}_{c}^{\mathrm{R} 2}$ and $\boldsymbol{p}_{e}^{\mathrm{R} 2}$ as shown in Fig. 9. Both the tip of the surgical instrument and its shadow will be visible in the microscopic view as long as the following inequality holds

$$
\theta_{\mathrm{C}_{1}, \mathrm{safe}} \geq \theta_{\mathrm{C}_{1}} \text {. }
$$

To integrate this into the linear constraints (23), we need to find Constraint (24) with respect to the joint velocities $\boldsymbol{q}$. Since $\boldsymbol{t}_{\mathrm{R} 1}^{\mathrm{R} 2}, \boldsymbol{p}_{c}^{\mathrm{R} 2}$ and $\boldsymbol{p}_{e}^{\mathrm{R} 2}$ are the functions of $\boldsymbol{q}_{\mathrm{R} 1}$ and $\boldsymbol{q}_{\mathrm{R} 2}$, we propose the following equivalent constraint

$$
\begin{gathered}
\theta_{\mathrm{C}_{1}, \text { safe }} \geq \theta_{\mathrm{C}_{1}} \Longleftrightarrow \\
\cos ^{2} \theta_{\mathrm{C}_{1}}-\cos ^{2} \theta_{\mathrm{C}_{1}, \text { safe }} \geq 0 \Longleftrightarrow \\
\left\|\boldsymbol{p}_{c}^{\mathrm{R} 2}\right\|^{2}\left\|\boldsymbol{t}_{\mathrm{R} 1}^{\mathrm{R} 2}\right\|^{2}\left\|\boldsymbol{p}_{e}^{\mathrm{R} 2}\right\|^{2}\left(\cos ^{2} \theta_{\mathrm{C}_{1}}-\cos ^{2} \theta_{\mathrm{C}_{1}, \text { safe }}\right) \geq 0 .
\end{gathered}
$$

We multiplied both sides of (25) by $\left\|p_{c}^{\mathrm{R} 2}\right\|^{2}\left\|\boldsymbol{t}_{\mathrm{R} 1}^{\mathrm{R} 2}\right\|^{2}\left\|\boldsymbol{p}_{e}^{\mathrm{R} 2}\right\|^{2}$. We use squared values to make sure that the time derivatives of the distance functions, which we need in Subsection IX-C, are defined everywhere. Finally, we can remove the explicit dependency on $\theta_{\mathrm{C}_{1}}$ and $\theta_{\mathrm{C}_{1} \text {, safe }}$ with the dot product between pure quaternions [31, Eq. (2)]

$$
\begin{aligned}
\left\langle\boldsymbol{p}_{c}^{\mathrm{R} 2}, \boldsymbol{t}_{\mathrm{R} 1}^{\mathrm{R} 2}\right\rangle & =\left\|\boldsymbol{p}_{c}^{\mathrm{R} 2}\right\|\left\|\boldsymbol{t}_{\mathrm{R} 1}^{\mathrm{R} 2}\right\| \cos \theta_{\mathrm{C}_{1}} \\
\left\langle\boldsymbol{p}_{c}^{\mathrm{R} 2}, \boldsymbol{p}_{e}^{\mathrm{R} 2}\right\rangle & =\left\|\boldsymbol{p}_{c}^{\mathrm{R} 2}\right\|\left\|\boldsymbol{p}_{e}^{\mathrm{R} 2}\right\| \cos \theta_{\mathrm{C}_{1}, \text { safe }} .
\end{aligned}
$$

By rearranging Constraint (25) with (26) and (27), we get $d_{\mathrm{C}_{1}}$ and $d_{\mathrm{C}_{1} \text {, safe }}$ as follows,

$$
\begin{gathered}
\left\|\boldsymbol{p}_{c}^{\mathrm{R} 2}\right\|^{2}\left\|\boldsymbol{t}_{\mathrm{R} 1}^{\mathrm{R} 2}\right\|^{2}\left\|\boldsymbol{p}_{e}^{\mathrm{R} 2}\right\|^{2}\left(\cos ^{2} \theta_{\mathrm{C}_{1}}-\cos ^{2} \theta_{\mathrm{C}_{1}, \text { safe }}\right) \geq 0 \Longleftrightarrow \\
\underbrace{\left\|\boldsymbol{p}_{e}^{\mathrm{R} 2}\right\|^{2}\left(\left\langle\boldsymbol{p}_{c}^{\mathrm{R} 2}, \boldsymbol{t}_{\mathrm{R} 1}^{\mathrm{R} 2}\right\rangle\right)^{2}}_{d_{\mathrm{C}_{1}}}-\underbrace{\left\|\boldsymbol{t}_{\mathrm{R} 1}^{\mathrm{R} 2}\right\|^{2}\left(\left\langle\boldsymbol{p}_{c}^{\mathrm{R} 2}, \boldsymbol{p}_{e}^{\mathrm{R} 2}\right\rangle\right)^{2}}_{d_{\mathrm{C}_{1}, \text { safe }}} \geq 0 .
\end{gathered}
$$

Note that $\boldsymbol{t}_{\mathrm{R} 1}^{\mathrm{R} 2}$ and $\boldsymbol{p}_{c}^{\mathrm{R} 2}$ can be trivially found as

$$
\boldsymbol{t}_{\mathrm{R} 1}^{\mathrm{R} 2}=\boldsymbol{t}_{\mathrm{R} 1}-\boldsymbol{t}_{\mathrm{R} 2} \quad \boldsymbol{p}_{c}^{\mathrm{R} 2}=\boldsymbol{p}_{c}-\boldsymbol{t}_{\mathrm{R} 2} .
$$

However, finding $\boldsymbol{p}_{e}^{\mathrm{R} 2}$ requires some geometrical reasoning, as explained in the following Section.

\section{B. How to find the edge point}

To find $\boldsymbol{p}_{e}^{\mathrm{R} 2}$ as a function of $\boldsymbol{q}_{\mathrm{R} 2}$ and $\boldsymbol{q}_{\mathrm{R} 1}$, let $\boldsymbol{p}_{a}^{\mathrm{R} 2}\left(\boldsymbol{q}_{\mathrm{R} 2}\right) \triangleq$ $\boldsymbol{p}_{a}^{\mathrm{R} 2}$ be the point in $\boldsymbol{p}_{c}^{\mathrm{R} 2}$ with the same height ( $z$-axis coordinate value) as $t_{\mathrm{R} 1}^{\mathrm{R} 2}$. Then, the following relationship holds

$$
\left|\left\langle\boldsymbol{p}_{a}^{\mathrm{R} 2}, \hat{k}\right\rangle\right|=\left|\left\langle\boldsymbol{t}_{\mathrm{R} 1}^{\mathrm{R}}, \hat{k}\right\rangle\right| \text {. }
$$

In addition, let $\phi \in \mathbb{R}$ be the angle between $\boldsymbol{p}_{c}^{\mathrm{R} 2}$ and the vertical axis, $\hat{k}$, as shown in Fig. 9 . Since $\phi$ is also the angle between $\boldsymbol{p}_{a}^{\mathrm{R} 2}$ and the vertical axis, $\hat{k}$, the following holds

$$
\cos \phi=\frac{\left|\left\langle\boldsymbol{p}_{a}^{\mathrm{R} 2}, \hat{k}\right\rangle\right|}{\left\|\boldsymbol{p}_{a}^{\mathrm{R} 2}\right\|}=\frac{\left|\left\langle\boldsymbol{p}_{c}^{\mathrm{R} 2}, \hat{k}\right\rangle\right|}{\left\|\boldsymbol{p}_{c}^{\mathrm{R} 2}\right\|} .
$$

By substituting (30) into (31), we can find

$\cos \phi=\frac{\left|\left\langle\boldsymbol{t}_{\mathrm{R} 1}^{\mathrm{R} 2}, \hat{k}\right\rangle\right|}{\left\|\boldsymbol{p}_{a}^{\mathrm{R} 2}\right\|}=\frac{\left|\left\langle\boldsymbol{p}_{c}^{\mathrm{R} 2}, \hat{k}\right\rangle\right|}{\left\|\boldsymbol{p}_{c}^{\mathrm{R} 2}\right\|} \Longrightarrow \frac{\left\|\boldsymbol{p}_{a}^{\mathrm{R} 2}\right\|}{\left\|\boldsymbol{p}_{c}^{\mathrm{R} 2}\right\|}=\frac{\left\langle\boldsymbol{t}_{\mathrm{R} 1}^{\mathrm{R} 2}, \hat{k}\right\rangle}{\left\langle\boldsymbol{p}_{c}^{\mathrm{R} 2}, \hat{k}\right\rangle}$,

where we dropped the modulus operator in (32) because both inner products will always be negative, given our definition of $\mathcal{F}_{\mathrm{R} 2}$ and with the assumption that $\boldsymbol{t}_{\mathrm{R} 2}$ is higher than both $t_{\mathrm{R} 1}$ and $\boldsymbol{p}_{c}$ along the vertical axis ${ }^{5}$. This is reasonable requirement because, for the shadow of the surgical instrument to be projected onto the retina, we need that assumption to hold.

In addition, using the fact that (32) represents the ratio of the lengths of $\boldsymbol{p}_{a}^{\mathrm{R} 2}$ and $\boldsymbol{p}_{c}^{\mathrm{R} 2}$, we can find

$$
\boldsymbol{p}_{a}^{\mathrm{R} 2}=\frac{\left\|\boldsymbol{p}_{a}^{\mathrm{R} 2}\right\|}{\left\|\boldsymbol{p}_{c}^{\mathrm{R} 2}\right\|} \boldsymbol{p}_{c}^{\mathrm{R} 2}=\underbrace{\frac{\left\langle\boldsymbol{t}_{\mathrm{R} 1}^{\mathrm{R} 2}, \hat{k}\right\rangle}{\left\langle\boldsymbol{p}_{c}^{\mathrm{R} 2}, \hat{k}\right\rangle}}_{a_{1}} \boldsymbol{p}_{c}^{\mathrm{R} 2}=a_{1} \boldsymbol{p}_{c}^{\mathrm{R} 2} .
$$

Finally, from the fact that $\boldsymbol{p}_{e}^{\mathrm{R} 2}-\boldsymbol{p}_{c}^{\mathrm{R} 2}$ and $\boldsymbol{p}_{a}^{\mathrm{R} 2}-\boldsymbol{t}_{\mathrm{R} 1}^{\mathrm{R} 2}$ have the same direction, we find

$$
\begin{gathered}
\left(\boldsymbol{p}_{e}^{\mathrm{R} 2}-\boldsymbol{p}_{c}^{\mathrm{R} 2}\right)=\underbrace{\frac{\left(\boldsymbol{p}_{a}^{\mathrm{R} 2}-\boldsymbol{t}_{\mathrm{R} 1}^{\mathrm{R} 1}\right)}{\left\|\boldsymbol{p}_{a}^{\mathrm{R} 2}-\boldsymbol{t}_{\mathrm{R} 1}^{\mathrm{R} 1}\right\|}}_{\boldsymbol{a}_{2}} r_{\mathrm{ms}} \\
\Longleftrightarrow \boldsymbol{p}_{e}^{\mathrm{R} 2}=\boldsymbol{p}_{c}^{\mathrm{R} 2}+\boldsymbol{a}_{2} r_{\mathrm{ms}} .
\end{gathered}
$$

Remark 1. From (33), we can see that there is a singularity when $\left\langle\boldsymbol{p}_{c}^{\mathrm{R} 2}, \hat{k}\right\rangle=0$, that is, when the tip of the light guide is on the $x-y$ plane of the retina. However, this is prevented by the vitreoretinal task constraint $\mathrm{C}_{\mathrm{r}}$.

Remark 2. As for (34), there is a singularity when $\left\|\boldsymbol{p}_{a}^{\mathrm{R} 2}-\boldsymbol{t}_{\mathrm{R} 1}^{\mathrm{R}}\right\|=0$, which happens when the surgical instrument's tip, $t_{\mathrm{R} 1}$, is in the cone's central axis, the line that connects $\boldsymbol{p}_{c}$ and $\boldsymbol{t}_{\mathrm{R} 2}$. When we can not define $d_{\mathrm{C}_{1}}-d_{\mathrm{C}_{1} \text {, safe }}$ because of this singularity, we, instead, apply another signed distance calculated using $\left\|\boldsymbol{p}_{a}^{\mathrm{R} 2}-\boldsymbol{t}_{\mathrm{R} 1}^{\mathrm{R} 2}\right\|=10 \mu \mathrm{m}$.

\section{Distance Jacobian and safe distance Jacobian for $C_{1}$}

Now that we have found $d_{\mathrm{C}_{1}}$ and $d_{\mathrm{C}_{1} \text {, safe }}$, the next goal is to find the corresponding Jacobians $\boldsymbol{J}_{\mathrm{C}_{1}}, \boldsymbol{J}_{\mathrm{C}_{1} \text {, safe }}$. We can get them by finding the time derivatives of $d_{\mathrm{C}_{1}}$ and $d_{\mathrm{C}_{1} \text {, safe with }}$ respect to the joint velocities $\dot{\boldsymbol{q}}$. The time derivative of $d_{\mathrm{C}_{1}}$ is

$$
d_{\mathrm{C}_{1}}=\overbrace{\left\|\boldsymbol{p}_{e}^{\mathrm{R} 2}\right\|^{2}}^{h_{1}} \overbrace{\left(\left\langle\boldsymbol{p}_{c}^{\mathrm{R} 2}, \boldsymbol{t}_{\mathrm{R} 1}^{\mathrm{R} 2}\right\rangle\right)^{2}}^{h_{2}} \Longrightarrow \dot{d}_{\mathrm{C}_{1}}=\dot{h}_{1} h_{2}+h_{1} \dot{h}_{2} .
$$

\footnotetext{
${ }^{5}$ These can be expressed mathematically as $\left.\left\langle\boldsymbol{t}_{\mathrm{R} 2}, \hat{k}\right\rangle\right\rangle\left\langle\boldsymbol{t}_{\mathrm{R} 1}, \hat{k}\right\rangle \Longleftrightarrow$ $\left\langle\boldsymbol{t}_{\mathrm{R} 1}^{\mathrm{R} 1}, \hat{k}\right\rangle\left\langle 0\right.$ and $\left.\left\langle\boldsymbol{t}_{\mathrm{R} 2}, \hat{k}\right\rangle\right\rangle\left\langle\boldsymbol{p}_{c}, \hat{k}\right\rangle \Longleftrightarrow\left\langle\boldsymbol{p}_{c}^{\mathrm{R} 2}, \hat{k}\right\rangle<0$, respectively.
} 
Analogously, the time derivative of $d_{\mathrm{C}_{1} \text {,safe }}$ is

$d_{\mathrm{C}_{1}, \mathrm{safe}}=\overbrace{\left\|\boldsymbol{t}_{\mathrm{R} 1}^{\mathrm{R} 2}\right\|^{2}}^{h_{3}} \overbrace{\left(\left\langle\boldsymbol{p}_{c}^{\mathrm{R} 2}, \boldsymbol{p}_{e}^{\mathrm{R} 2}\right\rangle\right)^{2}}^{h_{4}} \Longrightarrow \dot{d}_{\mathrm{C}_{1}, \text { safe }}=\dot{h}_{3} h_{4}+h_{3} \dot{h}_{4}$.

Moreover, we have (using the property [31, Eq. (4)])

$$
\dot{h}_{1}=2\left\langle\boldsymbol{p}_{e}^{\mathrm{R} 2}, \dot{\boldsymbol{p}}_{e}^{\mathrm{R} 2}\right\rangle \quad \dot{h}_{3}=2\left\langle\boldsymbol{t}_{\mathrm{R} 1}^{\mathrm{R}}, \dot{\boldsymbol{t}}_{\mathrm{R} 1}^{\mathrm{R} 2}\right\rangle
$$

and

$$
\begin{aligned}
& \dot{h}_{2}=\overbrace{2\left\langle\boldsymbol{p}_{c}^{\mathrm{R} 2}, \boldsymbol{t}_{\mathrm{R} 1}^{\mathrm{R} 2}\right\rangle}^{h_{5}}\left(\left\langle\boldsymbol{t}_{\mathrm{R} 1}^{\mathrm{R} 2}, \dot{\boldsymbol{p}}_{c}^{\mathrm{R} 2}\right\rangle+\left\langle\boldsymbol{p}_{c}^{\mathrm{R} 2}, \dot{\boldsymbol{t}}_{\mathrm{R} 1}^{\mathrm{R} 2}\right\rangle\right) \\
& \dot{h}_{4}=\underbrace{2\left\langle\boldsymbol{p}_{c}^{\mathrm{R} 2}, \boldsymbol{p}_{e}^{\mathrm{R} 2}\right\rangle}_{h_{6}}\left(\left\langle\boldsymbol{p}_{e}^{\mathrm{R} 2}, \dot{\boldsymbol{p}}_{c}^{\mathrm{R} 2}\right\rangle+\left\langle\boldsymbol{p}_{c}^{\mathrm{R} 2}, \dot{\boldsymbol{p}}_{e}^{\mathrm{R} 2}\right\rangle\right) .
\end{aligned}
$$

Then, we have to find $\dot{\boldsymbol{t}}_{\mathrm{R} 1}^{\mathrm{R} 2}, \dot{\boldsymbol{p}}_{c}^{\mathrm{R} 2}$, and $\dot{\boldsymbol{p}}_{e}^{\mathrm{R} 2}$ with respect to $\dot{\boldsymbol{q}}$. From (29), and by re-writing with vectors, we have ${ }^{6}$ (10) and

$$
\mathrm{v}_{4}\left(\dot{\boldsymbol{p}}_{c}^{\mathrm{R} 2}\right)=\mathrm{v}_{4}\left(-\dot{\boldsymbol{t}}_{\mathrm{R} 2}\right)=\underbrace{\left[\begin{array}{ll}
\boldsymbol{O}_{4 \times n_{1}}-\boldsymbol{J}_{t_{2}}
\end{array}\right]}_{\boldsymbol{J}_{c}^{\mathrm{R} 2}} \dot{\boldsymbol{q}} .
$$

Lastly, we find $\dot{\boldsymbol{p}}_{e}^{\mathrm{R} 2}$ from (34)

$$
\dot{\boldsymbol{p}}_{e}^{\mathrm{R} 2}=\dot{\boldsymbol{p}}_{c}^{\mathrm{R} 2}+\dot{\boldsymbol{a}}_{2} r_{\mathrm{ms}} .
$$

Using the quotient rule, we find

$$
\dot{\boldsymbol{a}}_{2}=\frac{d}{d t}(\underbrace{\overbrace{\boldsymbol{p}_{a}^{\mathrm{R} 2}-\boldsymbol{t}_{\mathrm{R} 1}^{\mathrm{R} 2}}^{\boldsymbol{h}_{7}}}_{\left\|\boldsymbol{h}_{7}\right\|})=\frac{\dot{\boldsymbol{h}}_{7}}{\left\|\boldsymbol{h}_{7}\right\|}-\frac{\boldsymbol{h}_{7} \frac{d\left(\left\|\boldsymbol{h}_{7}\right\|\right)}{d t}}{\left\|\boldsymbol{h}_{7}\right\|^{2}} .
$$

Then (using the property [39, Eq. (3)]), we have

$$
\frac{d}{d t}\left(\left\|\boldsymbol{h}_{7}\right\|\right)=\frac{\left\langle\boldsymbol{h}_{7}, \dot{\boldsymbol{h}}_{7}\right\rangle}{\left\|\boldsymbol{h}_{7}\right\|} \quad \dot{\boldsymbol{h}}_{7}=\dot{\boldsymbol{p}}_{a}^{\mathrm{R} 2}-\dot{\boldsymbol{t}}_{\mathrm{R} 1}^{\mathrm{R}} .
$$

We have found $\dot{\boldsymbol{t}}_{\mathrm{R} 1}^{\mathrm{R} 2}$ as a function of $\dot{\boldsymbol{q}}$ in (10). Then, the last derivative to be found is that of (33)

$$
\dot{\boldsymbol{p}}_{a}^{\mathrm{R} 2}=\dot{a}_{1} \boldsymbol{p}_{c}^{\mathrm{R} 2}+a_{1} \dot{\boldsymbol{p}}_{c}^{\mathrm{R} 2} ;
$$

where, using the quotient rule,

$$
\dot{a}_{1}=\frac{\left\langle\hat{k}, \dot{\boldsymbol{t}}_{\mathrm{R} 1}^{\mathrm{R} 2}\right\rangle}{\left\langle\boldsymbol{p}_{c}^{\mathrm{R} 2}, \hat{k}\right\rangle}-\frac{\left\langle\boldsymbol{t}_{\mathrm{R} 1}^{\mathrm{R} 2}, \hat{k}\right\rangle\left\langle\hat{k}, \dot{\boldsymbol{p}}_{c}^{\mathrm{R} 2}\right\rangle}{\left(\left\langle\boldsymbol{p}_{c}^{\mathrm{R} 2}, \hat{k}\right\rangle\right)^{2}} .
$$

As for (45), using the property [31, Eq. (2)] and from (10) and (40), we get

$$
\dot{a}_{1}=\underbrace{\mathrm{v}_{4}(\hat{k})^{T}\left\{\frac{\boldsymbol{J}_{\mathrm{R} 1}^{\mathrm{R} 2}}{\left\langle\boldsymbol{p}_{c}^{\mathrm{R} 2}, \hat{k}\right\rangle}-\frac{\left\langle\boldsymbol{t}_{\mathrm{R} 1}^{\mathrm{R} 1}, \hat{k}\right\rangle \boldsymbol{J}_{c}^{\mathrm{R} 2}}{\left(\left\langle\boldsymbol{p}_{c}^{\mathrm{R} 2}, \hat{k}\right\rangle\right)^{2}}\right\}}_{\boldsymbol{J}_{a_{1}}} \dot{\boldsymbol{q}} .
$$

${ }^{6} \boldsymbol{p}_{c}$ is constant, because the microscope does not move. Therefore, $\boldsymbol{p}_{c}=0$
We can now work backwards to find intermediate Jacobians that compose the distance Jacobians. Substituting (46) and (40) in (44) and noticing that $a_{1} \in \mathbb{R}, \boldsymbol{p}_{c}^{\mathrm{R} 2} \in \mathbb{H}_{p}$, we find

$$
\begin{gathered}
\mathrm{v}_{4}\left(\dot{\boldsymbol{p}}_{a}^{\mathrm{R} 2}\right)=\mathrm{v}_{4}\left(\boldsymbol{p}_{c}^{\mathrm{R} 2}\right) \dot{a}_{1}+a_{1} \mathrm{v}_{4}\left(\dot{\boldsymbol{p}}_{c}^{\mathrm{R} 2}\right) \\
=\underbrace{\left\{\mathrm{v}_{4}\left(\boldsymbol{p}_{c}^{\mathrm{R} 2}\right) \boldsymbol{J}_{a_{1}}+a_{1} \boldsymbol{J}_{c}^{\mathrm{R} 2}\right\}}_{\boldsymbol{J}_{a}^{\mathrm{R} 2}} \dot{\boldsymbol{q}}
\end{gathered}
$$

Substituting (47) and (10) in (43) results in

$$
\begin{gathered}
\mathrm{v}_{4}\left(\dot{\boldsymbol{h}}_{7}\right)=\overbrace{\left(\boldsymbol{J}_{a}^{\mathrm{R} 2}-\boldsymbol{J}_{\mathrm{R} 1}^{\mathrm{R} 2}\right)}^{\boldsymbol{J}_{h_{7}}} \dot{\boldsymbol{q}} \\
\frac{d}{d t}\left(\left\|\boldsymbol{h}_{7}\right\|\right)=\frac{\mathrm{v}_{4}\left(\boldsymbol{h}_{7}\right)^{T} \mathrm{v}_{4}\left(\dot{\boldsymbol{h}}_{7}\right)}{\left\|\boldsymbol{h}_{7}\right\|}=\overbrace{\frac{\mathrm{v}_{4}\left(\boldsymbol{h}_{7}\right)^{T} \boldsymbol{J}_{h_{7}}}{\left\|\boldsymbol{h}_{7}\right\|} \dot{\boldsymbol{q}}}^{\boldsymbol{J}_{\left\|h_{7}\right\|}}
\end{gathered}
$$

Moreover, substituting (48) and (49) in (42) results in

$$
\mathrm{v}_{4}\left(\dot{\boldsymbol{a}}_{2}\right)=\underbrace{\left(\frac{\boldsymbol{J}_{h_{7}}}{\left\|\boldsymbol{h}_{7}\right\|}-\frac{\mathrm{v}_{4}\left(\boldsymbol{h}_{7}\right) \boldsymbol{J}_{\left\|h_{7}\right\|}}{\left\|\boldsymbol{h}_{7}\right\|^{2}}\right)}_{\boldsymbol{J}_{a_{2}}} \dot{\boldsymbol{q}} .
$$

Finally, substituting (50) and (40) in (41), we get the last derivative to be found as

$$
\mathrm{v}_{4}\left(\dot{\boldsymbol{p}}_{e}^{\mathrm{R} 2}\right)=\overbrace{\left(\boldsymbol{J}_{c}^{\mathrm{R} 2}+r \boldsymbol{J}_{a_{2}}\right)}^{\boldsymbol{J}_{e}^{\mathrm{R} 2}} \dot{\boldsymbol{q}} .
$$

Then, using $\boldsymbol{J}_{\mathrm{R} 1}^{\mathrm{R} 2}, \boldsymbol{J}_{c}^{\mathrm{R} 2}, \boldsymbol{J}_{e}^{\mathrm{R} 2}$, we get (37), (38) and (39) as

$$
\begin{gathered}
\dot{h}_{1}=\overbrace{2 \mathrm{v}_{4}\left(\boldsymbol{p}_{e}^{\mathrm{R} 2}\right)^{T} \boldsymbol{J}_{e}^{\mathrm{R} 2} \dot{\boldsymbol{q}} \quad \dot{h}_{3}=\overbrace{2 \mathrm{v}_{4}\left(\boldsymbol{t}_{\mathrm{R} 1}^{\mathrm{R} 2}\right)^{T} \boldsymbol{J}_{\mathrm{R} 1}^{\mathrm{R} 2}}^{\boldsymbol{J}_{h_{1}}} \dot{\boldsymbol{q}},}^{\boldsymbol{J}_{h_{3}}} \overbrace{\dot{h}_{2}=\overbrace{h_{5}\left\{\mathrm{v}_{4}\left(\boldsymbol{t}_{\mathrm{R} 1}^{\mathrm{R} 2}\right)^{T} \boldsymbol{J}_{c}^{\mathrm{R} 2}+\mathrm{v}_{4}\left(\boldsymbol{p}_{c}^{\mathrm{R} 2}\right)^{T} \boldsymbol{J}_{\mathrm{R} 1}^{\mathrm{R} 2}\right\}}^{\boldsymbol{J}_{h_{2}}}} \dot{\boldsymbol{q}}, \\
\dot{h}_{4}=\underbrace{h\{}_{\boldsymbol{J}_{h_{4}}\left\{\mathrm{v}_{4}\left(\boldsymbol{p}_{e}^{\mathrm{R} 2}\right)^{T} \boldsymbol{J}_{c}^{\mathrm{R} 2}+\mathrm{v}_{4}\left(\boldsymbol{p}_{c}^{\mathrm{R} 2}\right)^{T} \boldsymbol{J}_{e}^{\mathrm{R} 2}\right\}} \dot{\boldsymbol{q}} .
\end{gathered}
$$

In conclusion, we can find the distance Jacobian for $\mathrm{C}_{1}$ by substituting (52) and (53) in (35), as follows

$$
\dot{d}_{C 1}=\overbrace{\left(h_{2} \boldsymbol{J}_{h_{1}}+h_{1} \boldsymbol{J}_{h_{2}}\right)}^{\boldsymbol{J}_{\mathrm{C}_{1}}} \dot{\boldsymbol{q}} .
$$

Besides, we can find the safe distance Jacobian for $\mathrm{C}_{1}$ by substituting (52) and (54) in (36), as follows

$$
\dot{d}_{\mathrm{C}_{1}, \text { safe }}=\overbrace{\left(h_{4} \boldsymbol{J}_{h_{3}}+h_{3} \boldsymbol{J}_{h_{4}}\right)}^{\boldsymbol{J}_{\mathrm{C}_{1}, \text { safe }}} \dot{\boldsymbol{q}} .
$$

\section{Distance function and safe distance function for $C_{2}$}

The goal of this section is to find a distance function and a safe distance function to enforce $\mathrm{C}_{2}$. We define these distances similarly to what was done in Section IX-A. The distance function is related with the distance between the axis of the shaft of the light guide and the tip of the surgical instrument,

$$
d_{\mathrm{C}_{2}}\left(\boldsymbol{q}_{\mathrm{R} 1}, \boldsymbol{q}_{\mathrm{R} 2}\right) \triangleq d_{\mathrm{C}_{2}} \in \mathbb{R}^{+} \text {. }
$$


The safe distance function corresponds to the edge of the illumination volume of the light guide,

$$
d_{\mathrm{C}_{2}, \text { safe }}\left(\boldsymbol{q}_{\mathrm{R} 1}, \boldsymbol{q}_{\mathrm{R} 2}\right) \triangleq d_{\mathrm{C}_{2}, \text { safe }} \in \mathbb{R}^{+}
$$

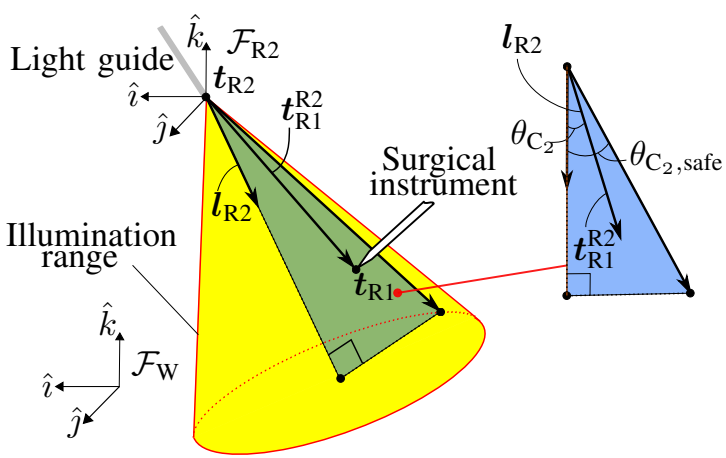

Fig. 10. The relationship between $\theta_{\mathrm{C}_{2}}$ and $\theta_{\mathrm{C}_{2}}$, safe used to define the distance

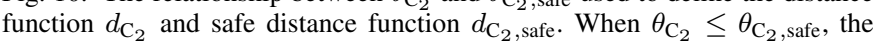
tip of the surgical instrument is kept inside the proposed cone.

In the following explanation, let the rotation of the light guide with respect to $\mathcal{F}_{\mathrm{W}}$ be the unit quaternion $\boldsymbol{r}_{\mathrm{R} 2}\left(\boldsymbol{q}_{\mathrm{R} 2}\right) \triangleq$ $\boldsymbol{r}_{\mathrm{R} 2} \in \mathbb{S}^{3}$. In addition, let the unit pure quaternion $\boldsymbol{l}_{\mathrm{R} 2}\left(\boldsymbol{q}_{\mathrm{R} 2}\right) \triangleq$ $l_{\mathrm{R} 2} \in \mathbb{H}_{p} \cap \mathbb{S}^{3}$ be the direction of the $z$-axis of the light guide (pointing outwards, collinear with the shaft of the light guide, the central axis of the illumination cone); that is,

$$
\boldsymbol{l}_{\mathrm{R} 2}=\boldsymbol{r}_{\mathrm{R} 2} \hat{k} \boldsymbol{r}_{\mathrm{R} 2}^{*} \text {. }
$$

With the above definitions, let $\theta_{\mathrm{C}_{2}}\left(\boldsymbol{q}_{\mathrm{R} 1}, \boldsymbol{q}_{\mathrm{R} 2}\right) \triangleq \theta_{\mathrm{C}_{2}} \in$ $[0, \pi / 2) \subset \mathbb{R}$ be the angle between $\boldsymbol{l}_{\mathrm{R} 2}$ and $\boldsymbol{t}_{\mathrm{R} 1}^{\mathrm{R} 2}$; and $\theta_{\mathrm{C}_{2} \text {, safe }}=$ $0.5 \mathrm{rad}$, which is constant in time because the illumination volume is inherent to the light guide, be the angle between $l_{\mathrm{R} 2}$ and the boundary of the illumination volume. The tip of the surgical instrument will always be in the illumination volume as long as the following constraint holds ${ }^{7}$

$$
\begin{gathered}
\theta_{\mathrm{C}_{2}, \text { safe }} \geq \theta_{\mathrm{C}_{2}} \Longleftrightarrow \\
\cos ^{2} \theta_{\mathrm{C}_{2}}-\cos ^{2} \theta_{\mathrm{C}_{2}, \text { safe }} \geq 0 \Longleftrightarrow \\
\left\|\boldsymbol{l}_{\mathrm{R} 2}\right\|^{2}\left\|\boldsymbol{t}_{\mathrm{R} 1}^{\mathrm{R} 2}\right\|^{2}\left(\cos ^{2} \theta_{\mathrm{C}_{2}}-\cos ^{2} \theta_{\mathrm{C}_{2}, \text { safe }}\right) \geq 0 .
\end{gathered}
$$

From the definition of the dot product, we have

$$
\left\langle\boldsymbol{l}_{\mathrm{R} 2}, \boldsymbol{t}_{\mathrm{R} 1}^{\mathrm{R} 2}\right\rangle=\left\|\boldsymbol{l}_{\mathrm{R} 2}\right\|\left\|\boldsymbol{t}_{\mathrm{R} 1}^{\mathrm{R} 2}\right\| \cos \theta_{\mathrm{C}_{2}} \text {. }
$$

By rearranging Constraint (60) with (61), we can remove the explicit dependency on $\theta_{\mathrm{C}_{2}}$, as follows

$$
\begin{aligned}
& \left\|\boldsymbol{l}_{\mathrm{R} 2}\right\|^{2}\left\|\boldsymbol{t}_{\mathrm{R} 1}^{\mathrm{R} 2}\right\|^{2}\left(\cos ^{2} \theta_{\mathrm{C}_{2}}-\cos ^{2} \theta_{\mathrm{C}_{2}, \text { safe }}\right) \geq 0 \Longleftrightarrow \\
& \underbrace{\left(\left\langle\boldsymbol{l}_{\mathrm{R} 2}, \boldsymbol{t}_{\mathrm{R} 1}^{\mathrm{R} 2}\right\rangle\right)^{2}}_{d_{\mathrm{C}_{2}}}-\underbrace{\left\|\boldsymbol{l}_{\mathrm{R} 2}\right\|^{2}\left\|\boldsymbol{t}_{\mathrm{R} 1}^{\mathrm{R} 2}\right\|^{2} \cos ^{2} \theta_{\mathrm{C}_{2}, \text { safe }}}_{d_{\mathrm{C}_{2}, \text { safe }}} \geq 0 .
\end{aligned}
$$

\footnotetext{
${ }^{7}$ Since $\theta_{\mathrm{C}_{2} \text {, safe }}$ is constant and does not depend on $\boldsymbol{q}_{\mathrm{R} 1}$ nor $\boldsymbol{q}_{\mathrm{R} 2}$, we do not need to remove the explicit dependency on $\theta_{\mathrm{C}_{2} \text {,safe }}$. Therefore, we multiply the following inequality with $\left\|\boldsymbol{l}_{\mathrm{R} 2}\right\|^{2}\left\|\boldsymbol{t}_{\mathrm{R} 1}^{\mathrm{R} 2}\right\|^{2}$ to get only the dot product (61).
}

\section{E. Distance Jacobian and safe distance Jacobian for $C_{2}$}

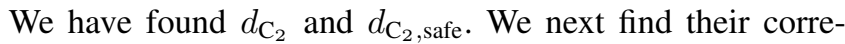

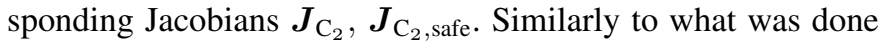
in Section IX-C, we can get them by finding the time derivatives of $d_{\mathrm{C}_{2}}$ and $d_{\mathrm{C}_{2} \text {, safe }}$ with respect to the joint velocities $\dot{\boldsymbol{q}}$. The time derivative of $d_{\mathrm{C}_{2}}$ is

$$
\begin{aligned}
d_{\mathrm{C}_{2}} & =\left(\left\langle\boldsymbol{l}_{\mathrm{R} 2}, \boldsymbol{t}_{\mathrm{R} 1}^{\mathrm{R} 2}\right\rangle\right)^{2} \\
\Longrightarrow \dot{d}_{\mathrm{C}_{2}} & =2\left\langle\boldsymbol{l}_{\mathrm{R} 2}, \boldsymbol{t}_{\mathrm{R} 1}^{\mathrm{R} 2}\right\rangle\left(\left\langle\boldsymbol{t}_{\mathrm{R} 1}^{\mathrm{R} 2}, \dot{\boldsymbol{l}}_{\mathrm{R} 2}\right\rangle+\left\langle\boldsymbol{l}_{\mathrm{R} 2}, \dot{\boldsymbol{t}}_{\mathrm{R} 1}^{\mathrm{R} 2}\right\rangle\right) .
\end{aligned}
$$

Besides, the time derivative of $d_{\mathrm{C}_{2} \text {, safe }}$ is

$$
\begin{aligned}
d_{\mathrm{C}_{2}, \text { safe }} & =\overbrace{\left\|\boldsymbol{l}_{\mathrm{R} 2}\right\|^{2}}^{h_{8}} \overbrace{\left\|\boldsymbol{t}_{\mathrm{R} 1}^{\mathrm{R} 2}\right\|^{2}}^{h_{9}} \cos ^{2} \theta_{\mathrm{C}_{2}, \text { safe }} \\
\Longrightarrow \dot{d}_{\mathrm{C}_{2}, \text { safe }} & =\left(\dot{h}_{8} h_{9}+h_{8} \dot{h}_{9}\right) \cos ^{2} \theta_{\mathrm{C}_{2}, \text { safe }} .
\end{aligned}
$$

As for $\dot{\boldsymbol{h}}_{8}, \dot{\boldsymbol{h}}_{9}$, using the property [31, Eq. (4)], we have

$$
\dot{h}_{8}=2\left\langle\boldsymbol{l}_{\mathrm{R} 2}, \dot{\boldsymbol{l}}_{\mathrm{R} 2}\right\rangle \quad \dot{h}_{9}=2\left\langle\boldsymbol{t}_{\mathrm{R} 1}^{\mathrm{R}}, \dot{\boldsymbol{t}}_{\mathrm{R} 1}^{\mathrm{R}}\right\rangle .
$$

Then, we need to find $\dot{\boldsymbol{l}}_{\mathrm{R} 2}$ and $\dot{\boldsymbol{t}}_{\mathrm{R} 1}^{\mathrm{R} 2}$ with respect to $\dot{\boldsymbol{q}}$. We have found $\dot{t}_{\mathrm{R} 1}^{\mathrm{R} 2}$ in (10). From (57), as we calculate $\mathrm{v}_{4}\left(\dot{\boldsymbol{l}}_{\mathrm{R} 1}\right)$ in (12), we obtain

$$
\begin{aligned}
\mathrm{v}_{4}\left(\dot{\boldsymbol{l}}_{\mathrm{R} 2}\right) & =\overbrace{\left(\begin{array}{ll}
\left.\overline{\boldsymbol{H}}_{4}\left(\hat{k} \boldsymbol{r}_{\mathrm{R} 2}^{*}\right)+\stackrel{+}{\boldsymbol{H}}_{4}\left(\boldsymbol{r}_{\mathrm{R} 2} \hat{k}\right) \boldsymbol{C}_{4}\right) \\
\boldsymbol{J}_{r_{2}}
\end{array} \dot{\boldsymbol{q}}_{\mathrm{R} 2}\right.}^{\underbrace{\boldsymbol{H}\left[\boldsymbol{O}_{4 \times n_{1}}\right.}_{\boldsymbol{J}_{\boldsymbol{l}_{\mathrm{R}}}} \boldsymbol{J}_{r_{2}}]} \dot{\boldsymbol{q}}
\end{aligned}
$$

where $\boldsymbol{J}_{r_{2}} \in \mathbb{R}^{4 \times n_{2}}$ is the rotation Jacobian of the robot R2.

We can now work backwards to find intermediate Jacobians that compose the distance Jacobians. Substituting (66) and (10) in (65) and the property [31, Eq. (2)] result in

$$
\begin{aligned}
& \dot{h}_{8}=\overbrace{2 \mathrm{v}_{4}\left(\boldsymbol{l}_{\mathrm{R} 2}\right)^{T} \boldsymbol{J}_{\boldsymbol{l}_{\mathrm{R} 2}}}^{\boldsymbol{J}_{h_{8}}} \dot{\boldsymbol{q}} \\
& \dot{h}_{9}=\underbrace{2 \mathrm{v}_{4}\left(\boldsymbol{t}_{\mathrm{R} 1}^{\mathrm{R} 2}\right)^{T} \boldsymbol{J}_{\mathrm{R} 1}^{\mathrm{R} 2}}_{\boldsymbol{J}_{h_{9}}} \dot{\boldsymbol{q}} .
\end{aligned}
$$

In conclusion, substituting (10) and (66) in (63) gives us the distance Jacobian for $\mathrm{C}_{2}$, as follows

$$
\dot{d}_{\mathrm{C}_{2}}=\overbrace{2\left\langle\boldsymbol{l}_{\mathrm{R} 2}, \boldsymbol{t}_{\mathrm{R} 1}^{\mathrm{R} 2}\right\rangle\left\{\mathrm{v}_{4}\left(\boldsymbol{t}_{\mathrm{R} 1}^{\mathrm{R} 2}\right)^{T} \boldsymbol{J}_{\boldsymbol{l}_{\mathrm{R} 2}}+\mathrm{v}_{4}\left(\boldsymbol{l}_{\mathrm{R} 2}\right)^{T} \boldsymbol{J}_{\mathrm{R} 1}^{\mathrm{R} 2}\right\}}^{\boldsymbol{J}_{\mathrm{C}_{2}}} \dot{\boldsymbol{q}} .
$$

Lastly, we can obtain the safe distance Jacobian for $\mathrm{C}_{2}$ by substituting (67) and (68) in (64), as follows

$$
\dot{d}_{\mathrm{C}_{2}, \text { safe }}=\overbrace{\left(h_{8} \boldsymbol{J}_{h_{9}}+h_{8} \boldsymbol{J}_{h_{9}}\right) \cos ^{2} \theta_{\mathrm{C}_{2}, \text { safe }}}^{\boldsymbol{J}_{\mathrm{C}_{2}, \text { safe }}} \dot{\boldsymbol{q}} .
$$




\section{Simulation AND EXPERIMENT}

We conducted three experiments and performed one simulation study to evaluate our proposed shadow-based autonomous positioning strategy.

First, in experiment E1, we evaluated the effectiveness and safety of our proposed conical constraints using a physical robotic system. Second, in simulation $\mathbf{S 1}$, we evaluated the autonomous instrument positioning in the entire retinal region using our three-step strategy. Third, in experiment E2, we evaluated a few representative points obtained in simulation S1. Lastly, in Experiment E3, we integrated our proposed autonomous control strategy with a data-driven image-processing algorithm and validated the fully integrated system.

\section{A. System configuration}

The simulations and experiments used the same software implementation on a Ubuntu 20.04 x64 system. The b-Cap protocol was used to communicate with the two robot arms (DENSO VS050, DENSO WAVE Inc., Japan) with a sampling frequency of $150 \mathrm{~Hz}$. Ros Noetic Ninjemys was used for the interprocess communication and CoppeliaSim (Coppelia Robotics, Switzerland) for the simulations. The dual quaternion algebra and robot kinematics were implemented using DQ Robotics [43] for Python3.

\section{B. Setup}

The physical robotic setup shown in Fig. 1 was used for the experiments. A simulated environment replicating this physical setup was implemented in CoppeliaSim.

A 7-mm-diameter retinal workspace (see Fig. 2) inside the Bionic-Eye was used. While performing the tasks described in the evaluation, the robot was commanded to maintain the shadow inside the workspace using the proposed conical constraints with $r_{\mathrm{ms}}=3.5 \mathrm{~mm}$.

Our focus in this work is to propose a safe and reliable robot control strategy for autonomous positioning on the retina. The image-processing aspect has been extensively addressed in related works [20], [44], and in this work, for simplicity, we use the Bionic-Eye with a white retinal background.

The instruments were initially positioned in such a way that all of the proposed constraints were satisfied (Premise III).

The calibration between elements in the workspace was performed once before the beginning of the trials. The calibration of each instrument's tip with respect to each robot was done through a pivoting process as often performed in the industry [31]. The calibration between the bases of each robot was conducted in a similar manner, using an image-based position tracker (Polaris Vega, NDI, Canada). The robots went through a high-accuracy calibration ${ }^{8}$. Using that information, the calibration between robots and the surgical phantom was performed using the kinematic models of the robots (Premise IV) and the positions of the insertion points described in Fig. 2. As routinely performed in robotic surgery, points $5 \mathrm{~mm}$

\footnotetext{
${ }^{8}$ This procedure is performed before the robots are shipped. The end-user has access to those parameters, but the details of the calibration procedure are patented [45]. Nonetheless, those parameters do not deviate much from their nominal values.
}

far from the tip are measured and marked. The instruments are then inserted into the Bionic-EyE until the mark matches the trocar points in the Bionic-Eye, by operating the robots' teaching pendants. Lastly, using the calculated positions of the insertion points and the relationship described in Fig. 2, we found the pose of the Bionic-EyE in the coordinate of the robot.

\section{Distance information in microscopic view}

Our strategy uses the distance information in the microscopic view to switch between steps in our three-step algorithm. Namely, we use the distance between the shadow's tip and the surgical instrument's shaft, $d_{\text {shaft }}$, for the overlap prevention step; and the distance between the shadow's tip and the surgical instrument's tip, $d_{\text {tip }}$, for the vertical positioning step.

In the first two experiments with the physical system, these distances were calculated geometrically, using only the kinematic information of the robots (Premise IV), and the thresholds $k_{\text {overlap }}$ and $k_{\text {vertical }}$ were set to $0.5 \mathrm{~mm}$ and $0.3 \mathrm{~mm}$, respectively ${ }^{9}$. In the final experiment, we used an image processing strategy to obtain these distances, and the thresholds $k_{\text {overlap }}$ and $k_{\text {vertical }}$ were set to $20 \mathrm{px}$ and 1 px, respectively. The values of the thresholds were determined for the movement of the instruments to appear natural in the microscopic image. Notice that we integrate our strategy with image processing as a feasibility study of the fully integrated system. There is no claim of novelty in the imaging-processing part, given that our autonomous control strategy can be integrated with any suitable imaging-processing strategy.

\section{Experiment E1: Evaluation of proposed conical constraints}

The first experiment was conducted to evaluate the conical VFIs we proposed for the visibility of the shadow.

In this experiment, the surgical instrument's tip was commanded to follow an arbitrarily predefined trajectory. The light guide was autonomously controlled by the proposed strategy. The instrument was moved in the plane, $\underline{\boldsymbol{\pi}}_{\text {planar }}$, described in Fig. (4), and the constrained optimization problem (4) was used. We defined the trajectory of the surgical instrument's tip as described in Fig. 11 so that the shadow gets outside the 7-mm-diameter workspace when no constraints are enforced.

We conducted the experiment under two conditions: with and without the proposed conical constraints. Then, we evaluated the behavior of the shadow in the microscopic view and the values of the conical constraints.

Results and discussion: The resulting trajectories are shown in Fig. 11. The trajectories depicted on the microscopic views were obtained by manually annotating the experimental videos. The trajectories calculated using the kinematic models are described next to the microscopic views. The controller succeeded in maintaining the tip of the shadow inside the workspace during the entire trial using the proposed conical constraints.

\footnotetext{
${ }^{9} \mathrm{We}$ set the thresholds so that the tip of the surgical instrument stopped before the tip touched and damaged the retina since image processing was not integrated in these experiments.
} 
(a) W/O Conical Constraints

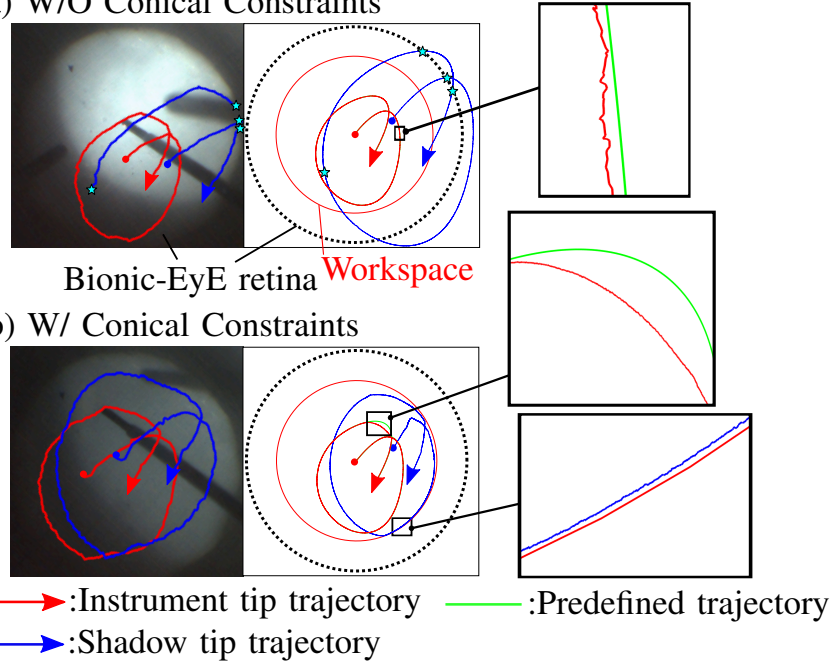

Fig. 11. The trajectory results of the evaluation of the proposed conical VFIs for the shadow visibility. (a) and (b) show the representative trajectories without and with the conical constraints, respectively. The controller succeeded in keeping the tip of the shadow inside the workspace. (a) Constraint $\mathrm{C}_{1}$

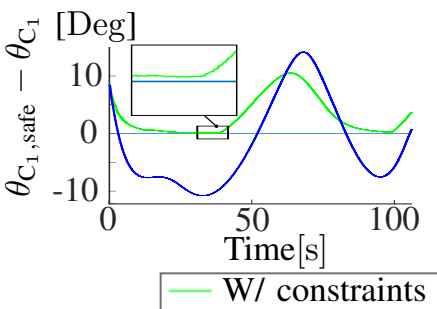

(b) Constraint $\mathrm{C}_{2}$

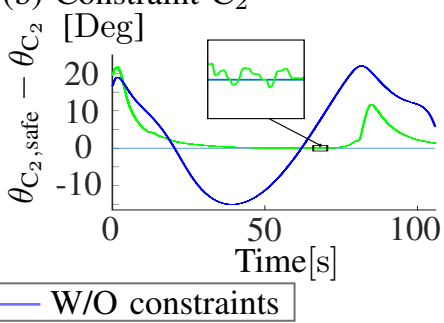

(c) Other constraints related to light guide maneuver [mm]

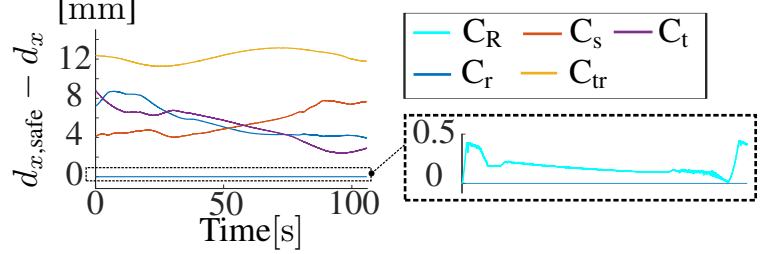

Fig. 12. The values related to the proposed constraints for the light guide. A negative value means the constraint is violated. (a) shows that the light guide satisfied the conical constraint $\mathrm{C}_{1}$ when it was autonomously controlled, and $\theta_{\mathrm{C}_{1}}$ and $\theta_{\mathrm{C}_{1} \text {, safe are introduced in (24). (b) shows that the conical }}$ constraint $\mathrm{C}_{2}$ had a negligible violation of at most $0.00014 \mathrm{rad}$ when it was autonomously controlled, and $\theta_{\mathrm{C}_{2}}$ and $\theta_{\mathrm{C}_{2} \text {, safe }}$ are introduced in (58). (c) shows that the light guide satisfied all other constraints when it was autonomously controlled, and the distances are introduced in Fig. 6.

Figs. 12-(a), (b) show the distances related to the conical constraints. A negative value means the constraint is violated. The conical constraint $\mathrm{C}_{1}$ was always non-negative, and $\mathrm{C}_{2}$ had a negligible violation of at most $0.0083 \mathrm{deg}(0.00014 \mathrm{rad})$.

Fig. 12-(c) shows the distances related to the constraints for the light guide maneuver (see Fig. 6). A negative value also means the constraint was violated. Fig. 12-(c) confirms that the light guide was autonomously controlled, satisfying all the constraints.

\section{E. Simulation S1: Evaluation of robustness}

We conducted a simulation study to assess the robustness of our shadow-based autonomous positioning strategy in the entire workspace. We tessellated the workspace into a grid of 700 points as described in Fig. 13 and evaluated whether the three-step algorithm converged properly on each step and was successful.

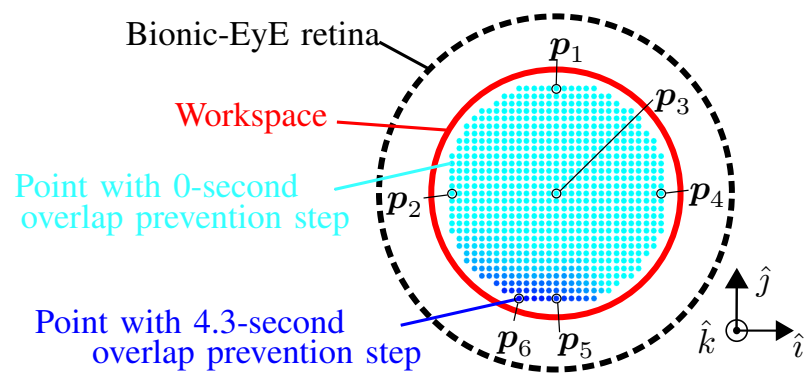

Fig. 13. The simulation result of our shadow-based autonomous positioning for 700 points inside the workspace. The coordinates are the same as in Fig. 2. The controller succeeded in positioning in all points. The color of each point represents the time needed to complete the overlap prevention step. Six points $\boldsymbol{p} \in\left(\boldsymbol{p}_{1}, \boldsymbol{p}_{2}, \boldsymbol{p}_{3}, \boldsymbol{p}_{4}, \boldsymbol{p}_{5}, \boldsymbol{p}_{6}\right)$ are the representative points used in the following experiments. $\boldsymbol{p}_{6}$ is the point with the longest time for the overlap prevention step.

Results and discussion: Our shadow-based autonomous positioning strategy properly converged and succeeded in positioning the surgical instrument's tip in all 700 points.

In Fig. 13, the color of each point represents the time needed for the overlap prevention step to converge. In fact, only some points around the lower-left part of the workspace needed the overlap prevention step. Because of the geometry of the eye and the trocar-point constraint, the instrument and its shadow tend to overlap near the insertion point of the light guide (see Fig. 2). The longest time needed to complete the overlap prevention step was approximately $4.3 \mathrm{~s}$ at most.

\section{F. Experiment E2: Evaluation using only the robot's kinematic models}

To evaluate our three-step shadow-based autonomous positioning strategy with the physical robotic system, we commanded the robot to move to representative points in the retina. We chose the six points $\boldsymbol{p} \in\left(\boldsymbol{p}_{1}, \boldsymbol{p}_{2}, \boldsymbol{p}_{3}, \boldsymbol{p}_{4}, \boldsymbol{p}_{5}, \boldsymbol{p}_{6}\right)$ described in Fig. 13, including the point that needed the most iterations to complete the overlap prevention step in the simulation study, $\boldsymbol{p}_{6}$. (a) Positioning process
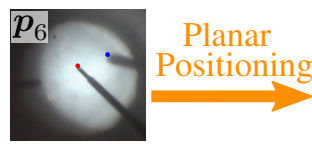

Overlap Prevention
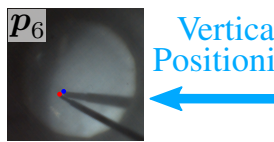
Positionin

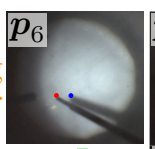

$p_{6}$

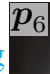

(b) Positioning results

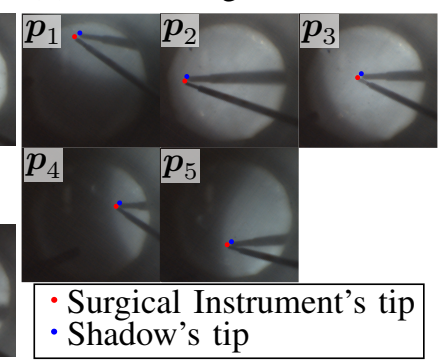

Fig. 14. The experimental results of the evaluation of the entire process of our shadow-based positioning method. (a) shows how the relative position of the surgical instrument and its shadow changed in the microscopic view. (b) shows the appearances after the proposed positioning to the representative points on the Bionic-EyE retina. 
Results and discussion: Fig. 14-(a) shows how the shadow behaved in the microscopic view through the entire shadowbased autonomous positioning process for $\boldsymbol{p}_{6}$. We can see that the overlap prevention step made the shadow easier to distinguish by separating it from the shaft of the surgical instrument. In addition, the controller succeeded in maintaining the tip of the shadow inside the microscopic view during the whole process.

Fig. 14-(b) shows the microscopic view after the positioning process for the representative points, $\boldsymbol{p}$. At all points, it is shown that our proposed strategy and controller can guide the shadow to a position where the shadow's tip can be used as a cue to position the surgical instrument's tip on the retina.

\section{G. Experiment E3: Integration with image processing}

In the following experiment, we integrated our system with an image-processing strategy and re-evaluated our three-step shadow-based autonomous positioning. For this purpose, we modified the Bionic-Eye's retina to be able to detect the contact between the surgical instrument's tip and the retina, trained a data-driven single-shot UNet-based [46] instrument tracking strategy, and slightly modified the three-step positioning switching conditions based on that extra information. These three modifications are described in detail as follows.

1) Detection of contact: To detect the contact between the surgical instrument's tip and the retina, we modified the setup as shown in Fig. 15-(a). We used a stainless steel needle and covered the retina with an aluminum sheet. We connected those to an oscilloscope. We can confirm the contact as a change in the waveform on the oscilloscope.

We used a stainless steel needle in this experiment because the forceps used in the previous experiments did not conduct current. Moreover, we painted the retina white to reflect the color of the retina used in the other experiments.

2) Image processing: To obtain the distances $d_{\text {shaft }}$ and $d_{\text {tip }}$ in the microscopic view, we implemented a UNet-based image-processing strategy. As shown in Fig 15-(b), the microscopic images $(2048 \times 2048 \times 3)$ were obtained using a camera (STC-MCCM401U3V, Omron-Sentech, Japan). The images were resized to $512 \times 512 \times 3$ and input to a segmentation model based on UNet [46]. The output of the network is the three-class pixel-wise semantic segmentation of the input image, classified into background, surgical instrument, or shadow. Using the semantic segmented image, we use the edge points of the predicted shapes as the tips, and the line that connects the surgical instrument's tip and the leftmost point of the surgical instrument is defined as the shaft. Finally, using these positions, $d_{\text {shaft }}$ and $d_{\text {tip }}$ are calculated.

The segmentation model was implemented using a PyTorch library [47], using a ResNet-34 pre-trained on ImageNet as encoder. To specialize the network to our application, we collected 150 microscopic images and manually segmented them to create ground truth images as shown in Fig. 16. Furthermore, we used common augmentation strategies [48]: blur, additive noise, image compression, rotation, optical distortion, and random brightness, and contrast. Considering all augmentations, we had 9000 images for training, 500 images for validation, and 500 images for testing.
The network was trained using dice loss, Adam optimizer with a learning rate of 0.0001 , and batch size of 4 . We trained the network on a single NVIDIA GeForce RTX 3070 GPU. The dice loss and Intersection over Union (IoU) values on the test set were 0.0063 and 0.9875 , respectively. Fig. 17 shows representative examples of the semantic segmentation.

3) Integration of the three-step algorithm with image processing: When $d_{\text {tip }}$ equals $1 \mathrm{px}$ in the microscopic view during the vertical positioning step (see Section X-C), it means that the instrument's tip is almost at the retina, as shown in Fig 15(c). After reaching that point, we rely on the kinematic model to slowly move the surgical instrument's tip downward.

The distance between the surgical instrument's tip and the retina after the vertical positioning step, $d_{\text {rest }}$, can be calculated using the geometrical relationship described in Fig. 15-(c), as follows

$$
d_{\text {rest }}[\mu \mathrm{m}]=1[\mathrm{px}] \times \frac{d_{z}[\mu \mathrm{m}]}{d_{x y}[\mu \mathrm{m}]} \times \frac{1}{\text { convert }[\mathrm{px} / \mu \mathrm{m}]},
$$

where $d_{x y}$ and $d_{z}$ are, respectively, the $x y$ and $z$ components of the relative position of the light guide's tip and the instrument's tip. In addition, convert $=0.015[\mathrm{px} / \mu \mathrm{m}]$ is calculated using as reference the diameter of the 1-mm-needle in the microscopic image. Lastly, to ensure positioning, we move the instrument $d_{\text {add }}=d_{\text {rest }}+100 \mu \mathrm{m}$ further. We call this as additional positioning, for convenience.

4) Validation: As a proof-of-concept integration validation, we integrated our proposed control strategy with image processing and evaluated our shadow-based autonomous positioning. For this, we conducted autonomous positioning for the six representative points used in S1 and E2, $p \in$ $\left(\boldsymbol{p}_{1}, \boldsymbol{p}_{2}, \boldsymbol{p}_{3}, \boldsymbol{p}_{4}, \boldsymbol{p}_{5}, \boldsymbol{p}_{6}\right)$ on the retina. The positioning for each point was repeated five times.

TABLE I

VALUES OF $d_{\text {ADD }}[\mu \mathrm{m}]$ FOR EACH POINT IN EACH TRIAL

\begin{tabular}{|c|c|c|c|c|c|c|c|}
\hline & T1 & T2 & T3 & T4 & T5 & Mean & Std. Dev. \\
\hline \hline $\boldsymbol{p}_{1}$ & $246^{\dagger}$ & $244^{\dagger}$ & $241^{\triangleright}$ & $242^{\dagger}$ & $240^{\dagger}$ & 243 & 2 \\
\hline $\boldsymbol{p}_{2}$ & $281^{\dagger}$ & $286^{\triangleright}$ & $289^{\dagger}$ & $289^{\dagger}$ & $290^{\dagger}$ & 287 & 4 \\
\hline $\boldsymbol{p}_{3}$ & $229^{\dagger}$ & $230^{\dagger}$ & $230^{\dagger}$ & $230^{\dagger}$ & $239^{\triangleright}$ & 232 & 4 \\
\hline $\boldsymbol{p}_{4}$ & $229^{\dagger}$ & - & $238^{\triangleright}$ & - & $236^{\triangleright}$ & 234 & 5 \\
\hline $\boldsymbol{p}_{5}$ & $349^{\dagger}$ & $351^{\star}$ & $354^{\dagger}$ & $354^{\dagger}$ & $352^{\dagger}$ & 352 & 3 \\
\hline $\boldsymbol{p}_{6}$ & $392^{\dagger}$ & $366^{\star}$ & $406^{\dagger}$ & - & $408^{\dagger}$ & 393 & 19 \\
\hline
\end{tabular}

Contact with the retina was verified using the oscilloscope.

$\diamond$ No contact

$\star$ Contact during the vertical positioning step

$\dagger$ Contact during the additional positioning step

- Force-quit (technical problems related to the proof-of-concept integration, stopped manually for safety)

Results and Discussion: Table I shows the values of $d_{\text {add }}[\mu \mathrm{m}]$ and how the autonomous positioning finished for each point in each trial. In the trials with $\dagger$, the surgical instrument's tip properly touched the retina during the additional positioning step.

In the cases marked with $\diamond$, the tip did not touch the retina even after the additional positioning step. On the other hand, in cases marked with $\star$, the surgical instrument's tip touched the retina before the additional positioning step. This can be 

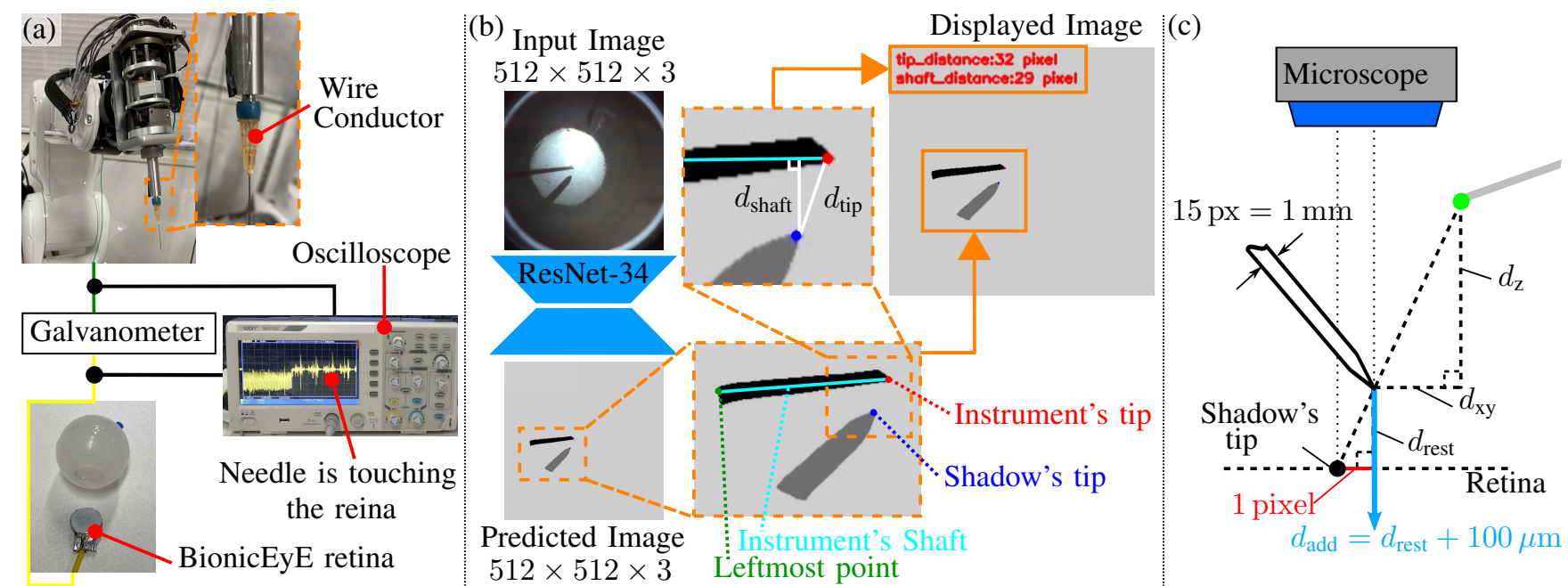

Fig. 15. Integration with image processing. (a) shows how we detect the contact between the surgical instrument's tip and the retina. The detection can be observed as the change of the waveform. (b) shows the flow of calculating the distances $d_{\text {shaft }}$ and $d_{\text {tip }}$ from the microscopic images. The shapes of the surgical instrument and the shadow are predicted using U-Net. Then, the distances are calculated based on the predicted image. (c) shows how much controller pushes the surgical instrument, $d_{\mathrm{add}}$, after vertical positioning step and the geometrical relation ship to calculate the distance.

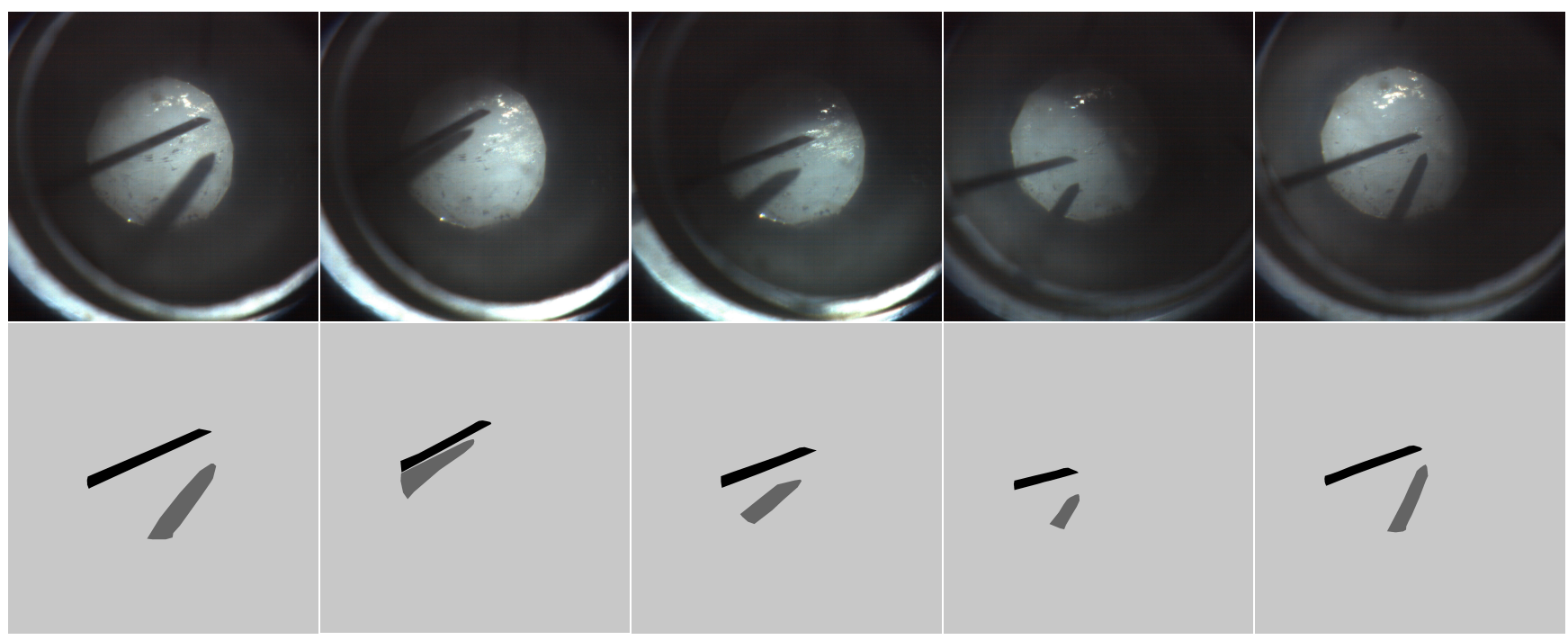

Fig. 16. Samples of manually segmented images.

caused by several factors, given that the scale of all objects in this experiment is very small. For instance, the surface of the retina that we prepared for this experiment was made to be convenient for detecting contact but does not have uniform height and placement. Moreover, the current resolution of the microscopic image is approximately $1 \mathrm{px}=70 \mu \mathrm{m}$ and must be improved in future work. This also affects the imageprocessing strategy to find the instrument's tip (e.g. the noise in Fig. 18), which should be further improved.

As for the cases of $\boldsymbol{p}_{4}$ with -, the needle used for this experiment was too short. A longer needle will be used in further validations.

Fig. 18 shows the system behavior during shadow-based autonomous positioning for $\boldsymbol{p}_{5}$. We can confirm that the controller switched between the steps according to the thresholds $k_{\text {overlap }}=20 \mathrm{px}$ and $k_{\text {vertical }}=1 \mathrm{px}$. The total computational time from acquiring the image to finishing calculating the distances was $0.093 \mathrm{~s}$. The distance $d_{\text {retina }}$ is calculated using the robot's kinematic parameters after the experiment.

\section{Conclusion}

In this work, a novel three-step shadow-based autonomous positioning strategy for vitreoretinal tasks was proposed. This method requires bimanual control that guarantees the visibility of the shadow of the surgical instrument's tip in the microscopic view. To achieve this, we derived new conical VFIs, used to autonomously move the light guide with respect to the surgical instrument. The results of the experimental and simulation studies confirm the feasibility of our positioning strategy in the entire workspace. In future work, we aim to improve image processing and use visual feedback for more accurate positioning. Furthermore, the proposed autonomous bimanual control of the light guide has the potential to improve the efficiency of surgical procedures and lead to new surgical 
Microscopic Image

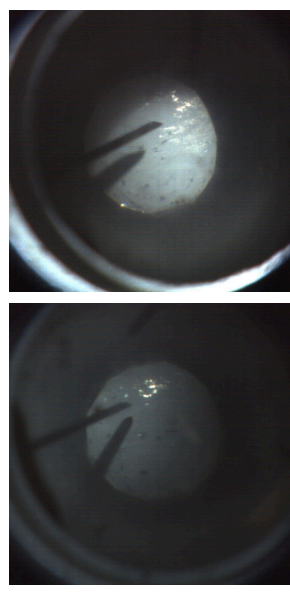

Ground Truth
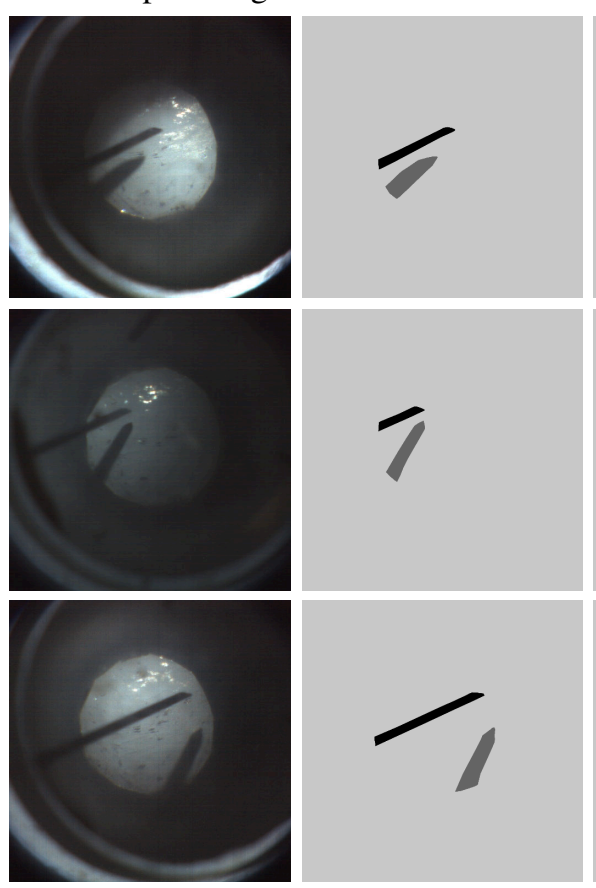

Fig. 17. Results of the semantic segmentation of three real microscopic images using the trained network.

techniques by setting the one hand of the surgeon free. We also aim to research how the proposed control can be used in collaborative work with the surgeons in future work.

\section{REFERENCES}

[1] S. Singh and C. Riviere, "Physiological tremor amplitude during retinal microsurgery," in Proceedings of the IEEE 28th Annual Northeast Bioengineering Conference. Philadelphia, PA, USA: IEEE, 2002, pp. $171-172$.

[2] R. Channa, I. Iordachita, and J. T. Handa, "Robotic Vitreoretinal Surgery:," Retina, vol. 37, no. 7, pp. 1220-1228, Jul. 2017.

[3] R. A. MacLachlan, B. C. Becker, J. C. Tabares, G. W. Podnar, L. A. Lobes, and C. N. Riviere, "Micron: An Actively Stabilized Handheld Tool for Microsurgery," IEEE Transactions on Robotics, vol. 28, no. 1, pp. 195-212, Feb. 2012.

[4] E. Kim, I. Choi, and S. Yang, "Design and Control of Fully Handheld Microsurgical Robot for Active Tremor Cancellation," in 2021 International Conference on Robotics and Automation (ICRA), Apr. 2021, p. 7.

[5] A. Uneri, M. A. Balicki, J. Handa, P. Gehlbach, R. H. Taylor, and I. Iordachita, "New steady-hand Eye Robot with micro-force sensing for vitreoretinal surgery," in 2010 3rd IEEE RAS \& EMBS International Conference on Biomedical Robotics and Biomechatronics. Tokyo, Japan: IEEE, Sep. 2010, pp. 814-819.

[6] A. Gijbels, J. Smits, L. Schoevaerdts, K. Willekens, E. B. Vander Poorten, P. Stalmans, and D. Reynaerts, "In-Human Robot-Assisted Retinal Vein Cannulation, A World First," Annals of Biomedical Engineering, vol. 46, no. 10, pp. 1676-1685, Oct. 2018.

[7] J. T. Wilson, M. J. Gerber, S. W. Prince, C.-W. Chen, S. D. Schwartz, J.-P. Hubschman, and T.-C. Tsao, "Intraocular robotic interventional surgical system (IRISS): Mechanical design, evaluation, and masterslave manipulation: Intraocular robotic interventional surgical system (IRISS)," The International Journal of Medical Robotics and Computer Assisted Surgery, vol. 14, no. 1, p. e1842, Feb. 2018.

[8] C.-Y. He, L. Huang, Y. Yang, Q.-F. Liang, and Y.-K. Li, "Research and Realization of a Master-Slave Robotic System for Retinal Vascular Bypass Surgery," Chinese Journal of Mechanical Engineering, vol. 31, no. 1, p. 78, Aug. 2018.
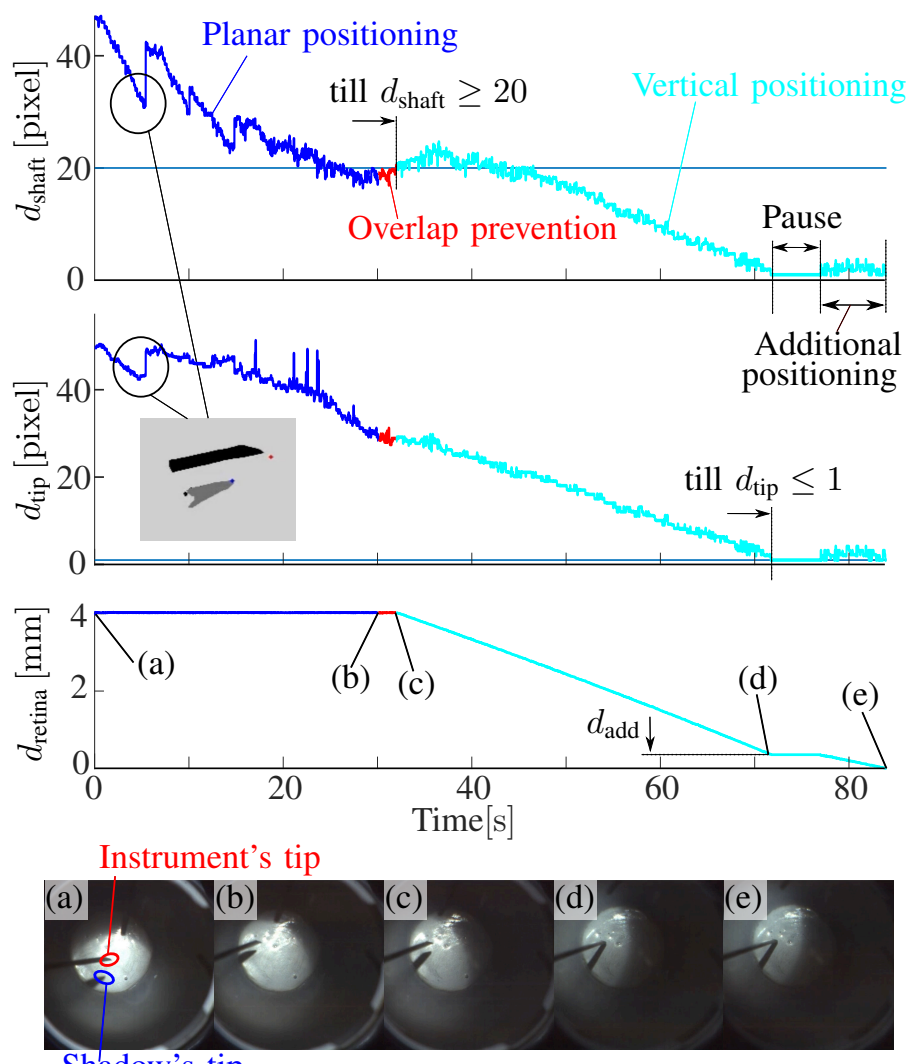

Shadow's tip

Fig. 18. The system behavior during shadow-based autonomous positioning for $\boldsymbol{p}_{5}$. The distance $d_{\text {retina }}$ is the distance between the surgical instrument's tip and the retina, calculated using the kinematic models of the robots, with zero at the end of positioning. The pictures below show the microscopic images at each stage.

[9] T. L. Edwards, K. Xue, H. C. M. Meenink, M. J. Beelen, G. J. L. Naus, M. P. Simunovic, M. Latasiewicz, A. D. Farmery, M. D. de Smet, and R. E. MacLaren, "First-in-human study of the safety and viability of intraocular robotic surgery," Nature Biomedical Engineering, vol. 2, no. 9, pp. 649-656, Sep. 2018.

[10] M. A. Nasseri, M. Eder, S. Nair, E. C. Dean, M. Maier, D. Zapp, C. P. Lohmann, and A. Knoll, "The introduction of a new robot for assistance in ophthalmic surgery," in 2013 35th Annual International Conference of the IEEE Engineering in Medicine and Biology Society (EMBC), Jul. 2013 , pp. 5682-5685.

[11] M. M. Marinho, K. Harada, A. Morita, and M. Mitsuishi, "SmartArm: Integration and validation of a versatile surgical robotic system for constrained workspaces," The International Journal of Medical Robotics and Computer Assisted Surgery, vol. 16, no. 2, Apr. 2020.

[12] S. Omata, Y. Someya, S. Adachi, T. Masuda, T. Hayakawa, K. Harada, M. Mitsuishi, K. Totsuka, F. Araki, M. Takao, M. Aihara, and F. Arai, "A surgical simulator for peeling the inner limiting membrane during wet conditions," PLOS ONE, vol. 13, no. 5, p. e0196131, May 2018.

[13] M. M. Marinho, B. V. Adorno, K. Harada, K. Deie, A. Deguet, P. Kazanzides, R. H. Taylor, and M. Mitsuishi, "A Unified Framework for the Teleoperation of Surgical Robots in Constrained Workspaces," in 2019 International Conference on Robotics and Automation (ICRA). Montreal, QC, Canada: IEEE, May 2019, pp. 2721-2727.

[14] M. M. Marinho, K. Harada, K. Deie, T. Ishimaru, and M. Mitsuishi, "SmartArm: Suturing Feasibility of a Surgical Robotic System on a Neonatal Chest Model," IEEE Transactions on Medical Robotics and Bionics, vol. 3, no. 1, pp. 253-256, Feb. 2021.

[15] Y. Tomiki, M. M. Marinho, Y. Kurose, K. Harada, and M. Mitsuishi, "On the use of general-purpose serial-link manipulators in eye surgery," in 2017 14th International Conference on Ubiquitous Robots and Ambient Intelligence (URAI), Jun. 2017, pp. 540-541.

[16] M. Ourak, "Combined OCT distance and FBG force sensing cannulation needle for retinal vein cannulation: In vivo animal validation," International Journal of Computer Assisted Radiology and Surgery, p. 9, 2019. 
[17] M. Zhou, M. Hamad, J. Weiss, A. Eslami, K. Huang, M. Maier, C. P. Lohmann, N. Navab, A. Knoll, and M. A. Nasseri, "Towards Robotic Eye Surgery: Marker-Free, Online Hand-Eye Calibration Using Optical Coherence Tomography Images," IEEE Robotics and Automation Letters, vol. 3, no. 4, pp. 3944-3951, Oct. 2018.

[18] M. Sommersperger, J. Weiss, M. Ali Nasseri, P. Gehlbach, I. Iordachita, and N. Navab, "Real-time tool to layer distance estimation for robotic subretinal injection using intraoperative 4D OCT," Biomedical Optics Express, vol. 12, no. 2, p. 1085, Feb. 2021.

[19] T. Tayama, Y. Kurose, M. M. Marinho, Y. Koyama, K. Harada, S. Omata, F. Arai, K. Sugimoto, F. Araki, K. Totsuka, M. Takao, M. Aihara, and M. Mitsuishi, "Autonomous Positioning of Eye Surgical Robot Using the Tool Shadow and Kalman Filtering," in 2018 40th Annual International Conference of the IEEE Engineering in Medicine and Biology Society $(E M B C)$. Honolulu, HI: IEEE, Jul. 2018, pp. 1723-1726.

[20] R. Richa, M. Balicki, R. Sznitman, E. Meisner, R. Taylor, and G. Hager, "Vision-Based Proximity Detection in Retinal Surgery," IEEE Transactions on Biomedical Engineering, vol. 59, no. 8, pp. 2291-2301, Aug. 2012.

[21] C. Bergeles, K. Shamaei, J. J. Abbott, and B. J. Nelson, "Single-Camera Focus-Based Localization of Intraocular Devices," IEEE Transactions on Biomedical Engineering, vol. 57, no. 8, pp. 2064-2074, Aug. 2010.

[22] C. Bergeles, B. E. Kratochvil, and B. J. Nelson, "Visually Servoing Magnetic Intraocular Microdevices," IEEE Transactions on Robotics, vol. 28, no. 4, pp. 798-809, Aug. 2012.

[23] S. Yang, J. N. Martel, L. A. Lobes, and C. N. Riviere, "Techniques for robot-aided intraocular surgery using monocular vision," The International Journal of Robotics Research, vol. 37, no. 8, pp. 931-952, Jul. 2018.

[24] A. Routray, R. A. MacLachlan, J. N. Martel, and C. N. Riviere, "RealTime Incremental Estimation of Retinal Surface Using Laser Aiming Beam," in 2019 International Symposium on Medical Robotics (ISMR). Atlanta, GA, USA: IEEE, Apr. 2019, pp. 1-5.

[25] J. W. Kim, C. He, M. Urias, P. Gehlbach, G. D. Hager, I. Iordachita, and M. Kobilarov, "Autonomously Navigating a Surgical Tool Inside the Eye by Learning from Demonstration," in 2020 IEEE International Conference on Robotics and Automation (ICRA), May 2020, pp. 73517357.

[26] M. Zhou, J. Wu, A. Ebrahimi, N. Patel, C. He, P. Gehlbach, R. H Taylor, A. Knoll, M. A. Nasseri, and I. Iordachita, "Spotlight-based 3D Instrument Guidance for Retinal Surgery," in 2020 International Symposium on Medical Robotics (ISMR). Atlanta, GA, USA: IEEE, Nov. 2020, pp. 69-75.

[27] B. C. Becker, R. A. MacLachlan, L. A. Lobes, and C. N. Riviere, "Semiautomated intraocular laser surgery using handheld instruments," Lasers in Surgery and Medicine, vol. 42, no. 3, pp. 264-273, Mar. 2010.

[28] J. Xia, S. J. Bergunder, D. Lin, Y. Yan, S. Lin, M. Ali Nasseri, M. Zhou, H. Lin, and K. Huang, "Microscope-Guided Autonomous Clear Corneal Incision," in 2020 IEEE International Conference on Robotics and Automation (ICRA). Paris, France: IEEE, May 2020, pp. 3867-3873.

[29] Wei Wei, R. Goldman, H. Fine, Stanley Chang, and N. Simaan, "Performance Evaluation for Multi-arm Manipulation of Hollow Suspended Organs," IEEE Transactions on Robotics, vol. 25, no. 1, pp. 147-157, Feb. 2009.

[30] C. He, E. Yang, N. Patel, A. Ebrahimi, M. Shahbazi, P. Gehlbach, and I. Iordachita, "Automatic Light Pipe Actuating System for Bimanual Robot-Assisted Retinal Surgery," IEEE/ASME Transactions on Mechatronics, vol. 25, no. 6, pp. 2846-2857, Dec. 2020.

[31] M. M. Marinho, B. V. Adorno, K. Harada, and M. Mitsuishi, "Dynamic Active Constraints for Surgical Robots Using Vector-Field Inequalities," IEEE Transactions on Robotics, vol. 35, no. 5, pp. 1166-1185, Oct. 2019.

[32] M. A. Nasseri, P. Gschirr, M. Eder, S. Nair, K. Kobuch, M. Maier, D. Zapp, C. Lohmann, and A. Knoll, "Virtual fixture control of a hybrid parallel-serial robot for assisting ophthalmic surgery: An experimental study," in 5th IEEE RAS/EMBS International Conference on Biomedical Robotics and Biomechatronics, Aug. 2014, pp. 732-738.

[33] Y. Yang, Z. Jiang, Y. Yang, X. Qi, Y. Hu, J. Du, B. Han, and G. Liu, "Safety Control Method of Robot-Assisted Cataract Surgery with Virtual Fixture and Virtual Force Feedback," Journal of Intelligent \& Robotic Systems, vol. 97, no. 1, pp. 17-32, Jan. 2020.

[34] T. Sakai, K. Harada, S. Tanaka, T. Ueta, Y. Noda, N. Sugita, and M. Mitsuishi, "Design and development of miniature parallel robot for eye surgery," in 2014 36th Annual International Conference of the IEEE Engineering in Medicine and Biology Society. Chicago, IL: IEEE, Aug. 2014, pp. 371-374.
[35] M. Yoshimura, M. M. Marinho, K. Harada, and M. Mitsuishi, "MBAPose: Mask and Bounding-Box Aware Pose Estimation of Surgical Instruments with Photorealistic Domain Randomization," p. 8.

[36] B. V. Adorno, Robot Kinematic Modeling and Control Based on Dual Quaternion Algebra - Part I: Fundamentals., Feb. 2017.

[37] V. M. Goncalves, P. Fraisse, A. Crosnier, and B. V. Adorno, "Parsimonious Kinematic Control of Highly Redundant Robots," IEEE Robotics and Automation Letters, vol. 1, no. 1, pp. 65-72, Jan. 2016.

[38] M. M. Marinho, H. Ishida, K. Harada, K. Deie, and M. Mitsuishi, "Virtual Fixture Assistance for Suturing in Robot-Aided Pediatric Endoscopic Surgery," IEEE Robotics and Automation Letters, vol. 5, no. 2, pp. 524-531, Apr. 2020.

[39] M. M. Marinho, B. V. Adorno, K. Harada, and M. Mitsuishi, "Active Constraints Using Vector Field Inequalities for Surgical Robots," in 2018 IEEE International Conference on Robotics and Automation (ICRA). Brisbane, QLD: IEEE, May 2018, pp. 5364-5371.

[40] O. Kanoun, F. Lamiraux, and P. Wieber, "Kinematic Control of Redundant Manipulators: Generalizing the Task-Priority Framework to Inequality Task," IEEE Transactions on Robotics, vol. 27, no. 4, pp. 785-792, Aug. 2011.

[41] F.-T. Cheng, T.-H. Chen, and Y.-Y. Sun, "Resolving manipulator redundancy under inequality constraints," IEEE Transactions on Robotics and Automation, vol. 10, no. 1, pp. 65-71, Feb. 1994.

[42] J. J. Quiroz-Omana and B. V. Adorno, "Whole-Body Control With (Self) Collision Avoidance Using Vector Field Inequalities," IEEE Robotics and Automation Letters, vol. 4, no. 4, pp. 4048-4053, Oct. 2019.

[43] B. V. Adorno and M. Marques Marinho, "DQ Robotics: A Library for Robot Modeling and Control," IEEE Robotics \& Automation Magazine, pp. 0-0, 2020.

[44] R. Richa, M. Balicki, E. Meisner, R. Sznitman, R. Taylor, and G. Hager, "Visual Tracking of Surgical Tools for Proximity Detection in Retinal Surgery," in Information Processing in Computer-Assisted Interventions, R. H. Taylor and G.-Z. Yang, Eds. Berlin, Heidelberg: Springer Berlin Heidelberg, 2011, vol. 6689, pp. 55-66.

[45] DENSO WAVE Inc., Japan, "Control system of a robot (in japanese)," Japan Patent p2017-061022, Mar 30, 2017. [Online]. Available: https://www.j-platpat.inpit.go.jp/c1800/PU/JP-2015-188521/ B1E5FAD41C196CE31D80C994582167F81A06DB57BE229BAE0FD। 2945EBE6B44C4/10/ja

[46] O. Ronneberger, P. Fischer, and T. Brox, "U-Net: Convolutional Networks for Biomedical Image Segmentation," arXiv:1505.04597 [cs], May 2015.

[47] P. Yakubovskiy, "Segmentation models pytorch," https://github.com/ qubvel/segmentation_models.pytorch, 2020.

[48] A. Buslaev, V. I. Iglovikov, E. Khvedchenya, A. Parinov, M. Druzhinin, and A. A. Kalinin, "Albumentations: Fast and flexible image augmentations," Information, vol. 11, no. 2, 2020. [Online]. Available: https://www.mdpi.com/2078-2489/11/2/125 